INSTITUT NATIONAL DE LA STATISTIQUE ET DES ETUDES ECONOMIQUES

Série des Documents de Travail du CREST

(Centre de Recherche en Economie et Statistique)

$n^{\circ}$ 2008-16

\title{
Optimal Litigation Strategies with Signaling and Screening*
}

\author{
Ph. CHONÉ \\ L. LINNEMER 2
}

Les documents de travail ne reflètent pas la position de l'INSEE et n'engagent que leurs auteurs.

Working papers do not reflect the position of INSEE but only the views of the authors.

\footnotetext{
* We are grateful to seminar participants at the 2007 CESifo Area Conference on Applied Microeconomics, the 22nd Meeting of the European Economic Association in Budapest, the 2007 ASSET Meeting in Padova as well as to Philippe Février and Thibaud Vergé for insightful comments.

${ }^{1}$ CREST-LEI, 28 rue des Saints-Pères, 75007 Paris. Email : chone@ensae.fr

2 CREST-LEI, 28 rue des Saints-Pères, 75007 Paris. Email : Laurent.linnemer@ensae.fr (contact author)
} 


\begin{abstract}
This paper examines the strategic effects of case preparation in litigation. Specifically, it shows how the pretrial efforts incurred by one party may alter its adversary's incentives to settle. We build a sequential game with one-sided asymmetric information where the informed party first decides to invest, or not, in case preparation, and the uninformed party then makes a settlement offer. Overinvestment, or bluff, always prevails in equilibrium: with positive probability, plaintiffs with weak cases take a chance on investing, and regret it in case of trial. Furthermore, due to the endogenous investment decision, the probability of trial may (locally) decrease with case strength. Overinvestment generates inefficient preparation costs, but may trigger more settlements, thereby reducing trial costs.
\end{abstract}

Keywords: Case preparation, Settlement, Trial, signaling.

JEL Classification: K410

\title{
Résumé
}

Cet article analyse les effets stratégiques de la préparation d'un cas lors d'une procédure judiciaire. Plus particulièrement, il montre que les efforts fournis par une partie lors de la phase préliminaire modifient les incitations de l'autre partie à proposer une transaction. L'article repose sur un jeu séquentiel où l'une des deux parties possède une information privée sur le cas. La partie informée investit ou pas dans la préparation du cas. L'autre partie observe cette décision et propose une transaction. À l'équilibre, il y a sur-investissement (bluff): avec une probabilité positive, les types avec des cas faibles investissent pour le regretter si l'affaire se termine par un procès. Par ailleurs, comme la décision d'investir est endogène, la probabilité de procès peut se révéler localement décroissante avec la force du cas. Il existe un arbitrage entre les coûts dus au sur-investissement et les économies réalisées lorsque le bluff conduit à plus de transactions.

Mots clefs: Préparation d'un cas, Transaction, Procès, Signal.

Classification du JEL : K410 


\section{Introduction}

The vast majority of tort disputes never reach a trial verdict. Litigants, indeed, have mutual incentives to save on trial costs by settling out of court. Moreover, a settlement shortens the dispute and might help to keep it confidential. ${ }^{1}$ For example, out of the 98,786 tort cases that were terminated in U.S. district courts during fiscal years 2002 and 2003, 1,647 or $2 \%$ were decided by a bench or jury trial. ${ }^{2}$ Data about settlement are most of the time not available but it is commonly believed that cases that go to trial involve larger damages. ${ }^{3}$ The amount at stake in a settlement dispute can be very important: in March 2006 the Canadian firm Research In Motion who manufactures the Blackberry email device agreed to pay a $\$ 612.5 \mathrm{~m}$ settlement amount to end a patent dispute with NTP Inc. a little known Virginia firm. ${ }^{4}$

In this article, we examine how the incentives to settle are modified when litigants can enhance the strength of their case by investing in case preparation during the pretrial phase. We assume that pretrial efforts incurred by the parties can change the probability that the defendant will be found liable at trial and/or the damage awarded to the plaintiff should liability be established. The seminal contributions in the field, Bebchuk (1984) and Reinganum and Wilde (1986), assume that the expected award is fixed, but known to one party only. The former paper considers a screening game: the uninformed party (the defendant, say) makes a settlement offer, which is rejected by plaintiffs with strong cases. The latter paper studies a signaling game: the informed party makes an offer which positively depends on the strength of his case, and the defendant refuses to pay a larger settlement amount with a higher probability. ${ }^{5}$

With few exceptions, the subsequent literature has treated the expected award in court as exogenous. Litigants, however, do invest in case preparation with the purpose of improving their position at trial and, consequently, at the negotiation table. During the pretrial phase, the parties take various actions: getting additional evidence, taking thorough initial interviews and depositions, obtaining statements from witnesses, issuing interrogatories, selecting expert witnesses, etc. In practice, the precise form of pretrial preparation depends on the legal procedure in force.

To show how the investment in case preparation of one party can affect its adversary's incentives to settle, we build a sequential game, where the informed party first decides to invest, or not, in case preparation, and the uninformed litigant, after observing this

\footnotetext{
${ }^{1}$ See Daughety and Reinganum (1999) for the issue of confidentiality.

${ }^{2}$ Source: Bureau of Justice Statistics Bulletin, August 2005, NCJ 208713. See http://www.ojp.usdoj . gov/bjs/pubalp2.htm\#Torts

${ }^{3}$ See Black, Silver, Hyman, and Sage (2005) and Chandra, Shantanu, and Seabury (2005). Kaplan, Sadka, and Silva-Mendez (2008) use a data set from labor tribunals in Mexico that provides information about settled cases as well as tried cases. They find that about $70 \%$ of lawsuits are settled, $15 \%$ dropped and $15 \%$ go to trial. They find, however, that plaintiffs that go to trial receive significantly lower final payments. They explain this difference by a selection effect as workers who exaggerate their claims settle less often, and may be punished in terms of final-payment amounts.

${ }^{4}$ The settlement, which was not easy to reach, ended four years of legal dispute in the U.S. between the two companies. Maybe the largest amounts that make newspapers front pages correspond to drug related civil action trials but they do not necessarily lead to the largest amounts per plaintiff.

${ }^{5}$ See Spier (2005) and Daughety and Reinganum (2005) for comprehensive surveys.
} 
decision, makes a take-it-or-leave-it settlement offer. ${ }^{6}$ We assume that case preparation efforts entail a sunk cost, which is incurred during the pretrial phase, and that they are effective in raising or reducing the expected award (depending on the party who invests). Conditionally on the investment decision, litigants play a screening game with a continuum of types à la Bebchuk, leading to settlement or trial. ${ }^{7}$ The endogenous investment decision, however, introduces a signaling dimension. The informed party can potentially use the investment to manipulate the defendant's beliefs and alter her incentives to settle.

The observability assumption is critical as it is the basis of the signaling mechanism. Admittedly, a party may not observe the exact amount of resources devoted by her adversary to prepare his case. At the very least, however, the counsel chosen by a litigant to assist him during the pretrial phase is known to the other party as counsels have many opportunities to interact during this phase. The counsel choice is a good indicator of case preparation expenses. Lawyer's fees vary substantially from one lawyer to another according to experience and reputation. For example, the Laffey Matrix ${ }^{8}$ allows an experienced federal court litigator to charge twice as much as a junior associate. Hiring a prominent law firm rather than an ordinary attorney is a major strategic decision, and this choice is public information before the settlement offers are made.

To present our main findings, we now suppose, for convenience, that the informed party is an injured plaintiff, and the uninformed party a potentially negligent defendant. Case preparation raises the value of the claim, but entails a sunk cost. We assume that, under symmetric information, only plaintiffs with strong cases do invest. For low expected damage types, the costs of case preparation exceed its return. In other words, the case preparation technology is tailored for plaintiffs with large damages.

Under asymmetric information, low-damage plaintiffs can mimic plaintiffs with more serious cases in the hope of a larger settlement offer. Such an incentive is well understood by the defendant. If total trial costs are relatively large, the mimicking incentive leads, through an unraveling process $\grave{a}$ la Akerloff, to full pooling: plaintiffs invest in case preparation irrespective of the strength of their case. As a result, an efficient technology is deserted.

If total trial costs are not too large, a more complex equilibrium pattern stands out. Plaintiffs with strong cases, who invest in case preparation under symmetric information, maintain this choice under asymmetric information. Plaintiffs with weak cases, who do not invest under symmetric information, however, are made indifferent between investing or not, and randomize between both options. When the defendant observes that the plaintiff has invested, she herself randomizes between a high and a low settlement offer. When she observes that he has not, she makes a deterministic low offer. Plaintiffs with strong cases reject all equilibrium settlement offers and proceed to trial. Plaintiffs with weak cases

\footnotetext{
${ }^{6}$ For a model with alternative offers, see Spier (1992).

${ }^{7}$ The informational asymmetry is one-sided. For models where both parties have private information, see Schweizer (1989) and Daughety and Reinganum (1994).

${ }^{8} \mathrm{~A}$ list of hourly rates (adapted each year to take into account inflation) for attorneys of varying experience levels prepared by the Civil Division of the United States Attorney's Office for the District of Columbia. This list is intended to be used in cases in which a fee-shifting statute permits the prevailing party to recover reasonable attorney's fees. See http://www.usdoj.gov/usao/dc/Divisions/Civil_ Division/Laffey_Matrix_4.html
} 
can be further distinguished with respect to their settlement strategy. Plaintiffs with very weak cases accept all equilibrium offers (whether they have invested or not), and earn an informational rent. Intermediate types settle if and only if they have invested and the defendant offers a large amount. That is, these types settle more often out of court if they invest than if they do not.

Overinvestment in case preparation is generic, and its extent is constant across equilibria. This phenomenon can be understood as bluff. Plaintiffs with intermediate types go to court with positive probability, and regret to have invested when a trial indeed takes place. High damage and low damage types, on the contrary, never regret their decision.

Furthermore, our model predicts that the probability of trial can decrease with the strength of the case. This is in sharp contrast with both Bebchuk and Reinganum and Wilde models, which predict that the probability of trial increases with the expected damages. Indeed, the more demanding the plaintiff, the less likely settlement occurs, otherwise all types of plaintiff would demand more. In our model, this logic fails because of a selection effect involving the plaintiffs with intermediate cases. In equilibrium, the larger their expected damage, the larger their probability of investment and, in turn, the larger their probability of settlement.

Overall, asymmetric information induces the relatively low types to overinvest in case preparation. This is socially inefficient as it increases the preparation sunk costs. Strategic effects, however, can mitigate this direct cost effect. Case preparation may indeed trigger more settlements for plaintiffs with intermediate cases, and consequently reduce trial costs.

Among the few papers that deal with case preparation and endogenize case strength, Hay (1995) is the closest to the present study. ${ }^{9}$ Hay, however, assumes away any exogenous heterogeneity concerning case strength, by supposing that discovery rules are able to eliminate any preexisting uncertainty. Accordingly, in the last stage of his game, plaintiffs differ only through their pretrial effort, which is not observed by the defendant. In other words, the final stage of Hay's game, which has a mixed strategy equilibrium, involves hidden action, but symmetric information. The mechanism is reminiscent to shirking models. The plaintiff would, under perfect information, work hard to prepare his case, and the defendant would make a substantial settlement offer. ${ }^{10}$ As a result of the hidden action problem, the plaintiff is tempted to shirk, and, in the equilibrium, both players resort to mixed strategies. Settlement fails when a low offer is made to a well-prepared plaintiff. Hay's assumption that discovery rules, in jurisdictions where such rules exist, eliminate any asymmetric information is extreme, because some information cannot be exchanged (voluntary or not) without parties incurring important costs. Parties may not even be aware of the existence of pieces of information that are relevant for the dispute. In contrast to Hay, we assume that some exogenous heterogeneity remains at the end of the pretrial phase, and that case preparation decisions are observed by the defendant. The two sets of assumptions are complementary.

Posey (1998) introduces the option for the plaintiff of either hiring an attorney at the beginning of the settlement process or delaying it until and unless the case proceeds to

\footnotetext{
${ }^{9}$ In Schrag (1999) the strength of a case is also endogenous through the discovery of evidence efforts undertaken by the parties. But the efforts are made after the strategic settlement phase that is studied.

${ }^{10} \mathrm{Such}$ a strategy is assumed to be better than sloppy preparation which would imply a meager settlement offer.
} 
trial. To hire a lawyer early can be used as a (costly) signal. Yet she assumes that the attorney costs are the same for low-damage and high-damage plaintiffs and that the presence of an attorney does not affect case strength. That is, Posey focuses on the cost aspect of the attorney exclusively: hiring an attorney early is a purely dissipative signal. Under asymmetric information and under the assumption that only contingency fee arrangements are available, she exhibits a counter intuitive separating equilibrium where the attorney is hired by the low damage claimant. ${ }^{11}$ Our approach complements hers. We focus on observable (hourly fee) arrangements, and we assume that the choice of a better case preparation technology is not dissipative, but is effective in strengthening the case.

A methodological contribution of this article is to provide a comprehensive analysis of a sequential signaling game with one-sided informational asymmetry, a continuum of types and a type-dependent reservation utility. The informed party, playing first, makes a binary decision (preparing or not), and the uninformed party replies with a continuous strategic variable (the settlement offer). Despite of the multiplicity of equilibria, we are able to show that important economic features (in particular, the extent of overinvestment) are constant across equilibria. We also demonstrate that the equilibria involve non-degenerated mixed strategies of both players. As already said, the main modeling difference with Hay (1995) is the presence of private information. Another difference is the timing of the game: sequential in the present paper, simultaneous in Hay. In contrast to the signaling game of Reinganum and Wilde (1986), both players, in our framework, resort to mixed strategies. Specifically, in their paper, the uninformed defendant randomizes between accepting or rejecting the settlement offer made by the informed plaintiff, while, in our model, no randomization takes place once a settlement offer is made as it is the informed party who accepts or rejects the offer. Here, the defendant randomizes between a generous and a conservative offer when the plaintiff opts for case preparation which induces the intermediate plaintiff types to accept or reject the offer. As to the plaintiff, he randomizes between investing, or not, in case preparation, using a probability that is not necessarily monotonic in case strength. Yet our model predicts a simple average pattern: above a critical threshold for case strength, all types invest and proceed to trial. Below the threshold, the average probability of investment depends on the fundamentals of the game (sunk and trial costs and effectiveness of case preparation) in a simple manner.

The paper is organized as follows. Section 2 presents the model. Section 3 details the strategies of the parties and presents some preliminary results. Section 4 characterizes the unique equilibrium when trial costs are relatively large, while section 5 deals with the relatively low trial cost case. Section 6 presents comparative statics and qualitative properties of the equilibria. Section 7 suggests alternative interpretations of the model and avenues for future research.

\footnotetext{
${ }^{11}$ When both contingency fee and hourly fee arrangements are available (but the arrangement choice is not observed by the defendant) there is no longer a separating equilibrium.
} 


\section{The model}

We consider a litigation framework with one-sided informational asymmetry. The expected award at trial, also referred to as "case strength", is the plaintiff's private information. The defendant only knows the distribution of case strength. The plaintiff can invest in case preparation to enhance his case. We posit a multiplicative effect: the investment multiplies the expected award at trial by a constant greater than one. Both litigants are risk neutral. ${ }^{12}$

\subsection{The litigation game}

The extensive form of the game is illustrated in Figure 1. Nature determines the plaintiff's type, noted $x$, according to a distribution $F$ with positive density $f$ on $[a, b]$. The plaintiff decides to invest in case preparation, which we note $e=H$, or to exert the basic level of effort, $e=L$. The investment involves a sunk (pretrial) cost $c>0$. The defendant, after observing the investment decision, makes a take-it-or-leave-it settlement offer. The plaintiff either accepts or refuses the offer. If the latter, the case goes to court, where the expected award to the plaintiff is $\mu x$ if he has invested, $x$ otherwise. The parameter $\mu>1$ is common knowledge. In other words, the return of the case preparation is a higher expected award at trial.

In addition to the sunk cost, the pretrial investment may alter the plaintiff's trial costs. The choice of a reputable attorney in the pretrial phase may imply larger trial cost as it might be costly to switch to a less expensive lawyer who would have to start from scratch Furthermore, case preparation can make the life of the opposite party in court harder, forcing it to incur higher costs at trial. Trial costs are noted $t_{H}^{D} \geq t_{L}^{D} \geq 0$ for the defendant and $t_{H}^{P} \geq t_{L}^{P} \geq 0$ for the plaintiff. Total trial costs in each situation are denoted $T_{L}=t_{L}^{P}+t_{L}^{D}$ and $T_{H}=t_{H}^{P}+t_{H}^{D}$. Negative expected claims are assumed away: the expected award in court is greater than the trial's costs for both technologies, even for the weakest case: $a>t_{L}^{P}$ and $\mu a>t_{H}^{P}+c$.

$\begin{array}{lllll}\begin{array}{l}\text { Nature } \\ \text { chooses } x\end{array} & \begin{array}{l}\text { The plaintiff } \\ \text { strengthens his } \\ \text { case or not }\end{array} & \begin{array}{l}\text { The defendant } \\ \text { offers a settle- } \\ \text { ment amount }\end{array} & \begin{array}{l}\text { The plaintiff ac- } \\ \text { cepts or refuses }\end{array} & \begin{array}{c}\text { Trial if no } \\ \text { settlement }\end{array} \\ \quad e \in\{H, L\} & s_{e} \geq 0, e \in\{H, L\} & A \text { or } R & \text { Payoffs }\end{array}$

Figure 1: Timeline of the game

Under symmetric information, litigants never go to court. The defendant observes $x$,

\footnotetext{
${ }^{12}$ The roles could be switched: the defendant could be the one holding private information and investing. In that case the investment would reduce the expected award of the plaintiff at trial. The uninformed plaintiff would be the one making the settlement offer. See Section 7.
} 
the case strength, as well as the plaintiff's investment decision. Therefore, she holds all the necessary information to make personalized offers which are accepted. Formally, she offers $x-t_{L}^{P}$ after $e=L$, and $\mu x-t_{H}^{P}$ after $e=H$. The plaintiff accepts such an amount (but would refuse any smaller amount) because this is exactly the expected amount he would have in case of a trial. Anticipating these settlement amounts, the plaintiff invests if and only if $\mu x-t_{H}^{P}-c \geq x-t_{L}^{P}$. Let $\widetilde{x}$ be the type of the plaintiff who is indifferent, under perfect information, between both technologies:

$$
\widetilde{x}=\frac{t_{H}^{P}-t_{L}^{P}+c}{\mu-1} .
$$

We refer to the plaintiff $\widetilde{x}$ as the marginal type. Investment is efficient for plaintiffs with strong cases $(x>\widetilde{x})$, while it is not for weak cases $(x<\widetilde{x})$. Throughout, we assume that no technology is superior to the other for all types of plaintiff. Formally:

Assumption 1. The marginal type is interior: $a<\widetilde{x}<b$.

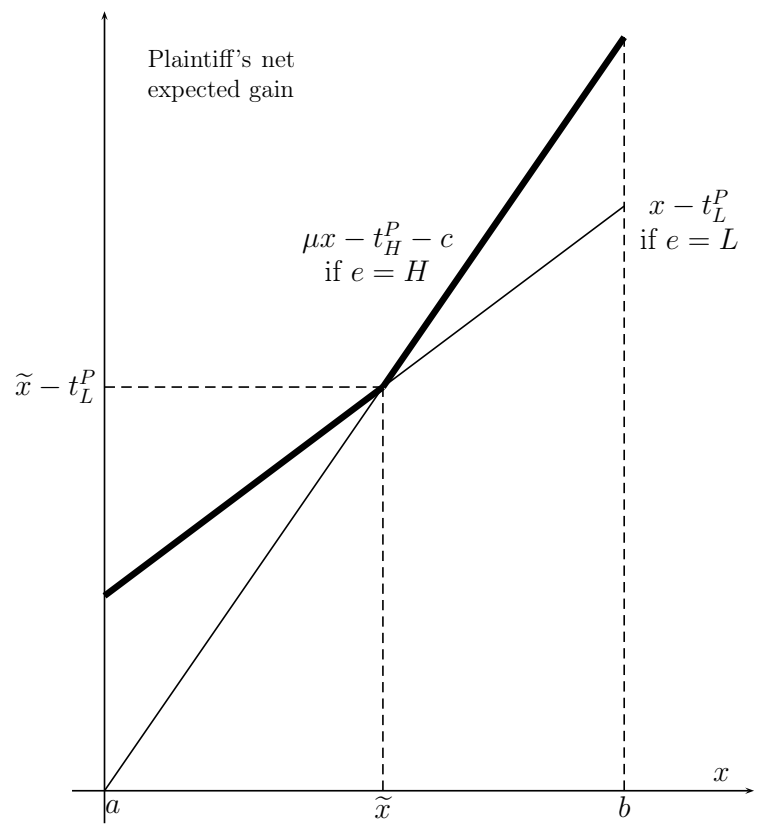

Figure 2: The efficient technologies and the marginal type $\widetilde{x}$

Under symmetric information, the plaintiff invests if and only if his case is strong $(x \geq$ $\widetilde{x})$, consequently he obtains $\mu x-t_{H}^{P}-c$. Otherwise he does not invest and gets $x-t_{L}^{P}$. In Figure 2, the bold line represents the plaintiff's symmetric information gain as a function of his type $x$. Under asymmetric information, this line corresponds to the minimum gain the plaintiff can secure by going to court. This gain is the plaintiff's reservation utility, which is type-dependent. We also assume that a plaintiff who has invested in case preparation does not want to switch back to the basic technology. 
Assumption 2. Once the sunk cost c has been incurred, a plaintiff has no incentives to give up the return of case preparation. Formally: $a-t_{L}^{P}<\mu a-t_{H}^{P}$.

Combined with Assumption 1, Assumption 2 implies, for the weakest case: $\mu a-t_{H}^{P}-c<$ $a-t_{L}^{P}<\mu a-t_{H}^{P}$, which entails a positive lower bound for the sunk cost: $c>\left(\mu a-t_{H}^{P}\right)-$ $\left(a-t_{L}^{P}\right)>0$. Notice that assumption 2 is satisfied when $t_{L}^{P}=t_{H}^{P}$.

\subsection{The one-technology benchmarks}

Throughout, we note $\{H\}$ and $\{L\}$ the situations where only one technology is available, and $\{H L\}$ the situation where the plaintiff can choose his preferred technology. Following Bebchuk, we first examine the benchmark cases $\{H\}$ and $\{L\}$.

A settlement offer partitions the population of plaintiffs into two groups. In case $\{L\}$, the plaintiff of type $x$ accepts a settlement offer $s$ if and only if $x \leq s+t_{L}^{P}$. The corresponding threshold in case $\{H\}$ is $\left(s+t_{H}^{P}\right) / \mu$. It is convenient to parameterize settlement offers with the type of the indifferent plaintiff, rather than with the settlement amount itself. The offer leaving plaintiff $x$ indifferent yields the following utility to plaintiff $y$ :

$$
\begin{aligned}
v_{\{L\}}(y ; x) & =\max \left(y-t_{L}^{P}, x-t_{L}^{P}\right) \\
v_{\{H\}}(y ; x) & =\max \left(\mu y-t_{H}^{P}-c, \mu x-t_{H}^{P}-c\right),
\end{aligned}
$$

and the following profit to the defendant:

$$
\begin{aligned}
\pi_{\{L\}}(x) & =-\left(x-T_{L}\right) F(x)-\int_{x}^{b} y f(y) \mathrm{d} y-t_{L}^{D} \\
\pi_{\{H\}}(x) & =\mu\left[-\left(x-\frac{T_{H}}{\mu}\right) F(x)-\int_{x}^{b} y f(y) \mathrm{d} y\right]-t_{H}^{D} .
\end{aligned}
$$

The latter formulae express the defendant's tradeoff between rent extraction and trial cost savings. Throughout the paper, we maintain the following assumption.

Assumption 3. The distribution of case strength is strictly log-concave.

Assumption 3 amounts to $\tau=F / f$ being increasing on $[a, b]$. Under this assumption, the function $\pi_{\{L\}}$ and $\pi_{\{H\}}$ are strictly quasi-concave. Therefore they attain their maximum for only one value of $x .{ }^{13}$ The optimal offers, denoted $x_{L}^{*}$ and $x_{H}^{*}$, are characterized by the first order conditions:

$$
\tau\left(x_{H}^{*}\right)=T_{H} / \mu \quad \text { and } \quad \tau\left(x_{L}^{*}\right)=T_{L} .
$$

If case strength is uniformly distributed on $[a, b]$, then $\tau(x)=x-a, x_{L}^{*}=a+T_{L}, x_{H}^{*}=$ $a+T_{H} / \mu$. By assumption, $T_{L} \leq T_{H}$, but $T_{H} / \mu$ could be either larger or smaller than $T_{L}$ and there is a priori no restriction on the ordering of $x_{H}^{*}$ and $x_{L}^{*}$. Hereafter, we limit our attention to the interesting cases where $x_{H}^{*}$ and $x_{L}^{*}$ are interior.

\footnotetext{
${ }^{13}$ In the three situations $\{H\},\{L\}$ and $\{H L\}$, existence results only require $\tau$ to be nondecreasing, but uniqueness results depend on $\tau$ increasing. In particular, the strict monotonicity guarantees the uniqueness of $x_{H}^{*}$ and $x_{L}^{*}$.
} 
In such a litigation environment, total welfare equals the opposite of litigation costs, and is not affected by transfers from one party to another. ${ }^{14}$ The expected litigation costs in equilibrium when only one technology is available are given by

$$
\mathcal{C}_{\{H\}}^{*}=c+\left(1-F\left(x_{H}^{*}\right)\right) T_{H} \quad \text { and } \quad \mathcal{C}_{\{L\}}^{*}=\left(1-F\left(x_{L}^{*}\right)\right) T_{L} .
$$

The comparison of the expected trial costs in the situations $\{H\}$ and $\{L\}$ involves a direct cost effect and a strategic effect. Formally,

$$
\mathcal{C}_{\{H\}}^{*}-\mathcal{C}_{\{L\}}^{*}=c+\left(1-F\left(x_{L}^{*}\right)\right)\left(T_{H}-T_{L}\right)-\left(F\left(x_{H}^{*}\right)-F\left(x_{L}^{*}\right)\right) T_{H} .
$$

Since $c \geq 0$ and $T_{H} \geq T_{L}$, the sum of the first two terms is positive, and tends to make $\mathcal{C}_{\{H\}}^{*}$ higher than $\mathcal{C}_{\{L\}}^{*}$ (direct cost effect). The last term reflects the change in the incentives to settle. If $T_{H} / \mu \leq T_{L}$, trial occurs less often in $\{L\}$ than in $\{H\}$, so both effects play in the same direction. This happens, in particular, when $T_{L}=T_{H}$. On the other hand, if $T_{H} / \mu$ is larger than $T_{L}$, the strategic effect tends to make $\mathcal{C}_{\{H\}}^{*}$ lower than $\mathcal{C}_{\{L\}}^{*}$. When $x_{H}^{*}$ tends to $b$, the strategic effect may dominate the direct cost effect. ${ }^{15}$

\section{Preliminary results}

We now examine the incentives to invest and to settle in the situation $\{H L\}$ where both technologies are available. As will shortly become clear, we must consider mixed strategies of the defendant. Parameterizing settlement offers with the type of the indifferent plaintiff as explained above, the most general defendant's strategy is represented by a pair $\left(P_{H}, P_{L}\right)$ of probability measures on the interval $[a, b]$. Facing $e=H$ (resp. $e=L$ ), the defendant randomizes between the offers $\mu x-t_{H}^{P}$ (resp. $x-t_{L}^{P}$ ), where $x$ is drawn in $[a, b]$ according to the distribution $P_{H}$ (resp. $P_{L}$ ).

\subsection{The defendant's strategy and the plaintiff's payoffs}

Facing a defendant's strategy $\left(P_{H}, P_{L}\right)$, a plaintiff of type $y$ gets the following expected payoffs:

$$
v_{H}(y)=\int_{a}^{b} v_{\{H\}}(y ; x) d P_{H}(x) \quad \text { and } \quad v_{L}(y)=\int_{a}^{b} v_{\{L\}}(y ; x) d P_{L}(x),
$$

where the base utility functions $v_{\{H\}}(. ; x)$ and $v_{\{L\}}(. ; x)$ are defined in (1) and (2).

Let $K_{L}$ be the set of nondecreasing, convex functions $v$ from $[a, b]$ to $\left[a-t_{L}^{P}, b-t_{L}^{P}\right]$ satisfying $v(b)=b-t_{L}^{P}$ and $0 \leq v^{\prime} \leq 1$. Similarly, let $K_{H}$ be the set of nondecreasing, convex functions $v$ from $[a, b]$ to $\left[\mu a-t_{H}^{P}-c, \mu b-t_{H}^{P}-c\right]$ satisfying $v(b)=\mu b-t_{H}^{P}-c$ and $0 \leq v^{\prime} \leq \mu$.

\footnotetext{
${ }^{14}$ The focus of the paper is on the settlement issue. Therefore we do not take into account the administrative costs of a trial nor the deterrence effects that trial and settlement cost might have.

${ }^{15}$ This is the case, for instance, when $T_{H} / \mu$ tends to $b-a$ and case strength is uniformly distributed.
} 
Lemma 1. There exists a one-to-one map between pairs $\left(P_{H}, P_{L}\right)$ of probability distributions on $[a, b]$ and pairs $\left(v_{H}, v_{L}\right) \in K_{H} \times K_{L}$. Conditionally on the litigation technologies, the trial probabilities are given by:

$$
P_{H}(x \leq y)=v_{H}^{\prime}(y) / \mu \text { and } P_{L}(x \leq y)=v_{L}^{\prime}(y)
$$

and are nondecreasing in case strength.

Proof. For all $x \in[a, b]$, the functions $v_{\{H\}}(. ; x)$ and $v_{\{L\}}(. ; x)$ belong to $K_{H}$ and $K_{L}$ respectively. Both sets are convex. The functions $v_{H}$ and $v_{L}$ given by (4) being convex combinations of the base functions $v_{\{H\}}(. ; x)$ and $v_{\{L\}}(. ; x)$, also belong to $K_{H}$ and $K_{L}$. We have:

$$
\begin{aligned}
& v_{H}(y)=\left[\mu y-t_{H}^{P}\right] P_{H}(x \leq y)+\int_{y}^{b}\left[\mu x-t_{H}^{P}\right] \mathrm{d} P_{L}(x)-c \\
& v_{L}(y)=\left[y-t_{L}^{P}\right] P_{L}(x \leq y)+\int_{y}^{b}\left[x-t_{L}^{P}\right] \mathrm{d} P_{L}(x)
\end{aligned}
$$

where $P_{e}(x \leq y)$ is the probability that the plaintiff of type $y$, if he exerts effort $e$, goes to trial. We refer to this probability as the conditional trial probability. Being nondecreasing and convex, $v_{e}$ is differentiable almost everywhere. ${ }^{16}$ Differentiating (6) and (7) yields (5). Since $v_{L}$ and $v_{H}$ are convex, the conditional trial probabilities are nondecreasing in case strength.

In Appendix A, we show that the base functions $v_{\{H\}}(. ; x)$ and $v_{\{L\}}(. ; x), a \leq x \leq b$, generate the convex sets $K_{H}$ and $K_{L}$ and explain how any function $v_{e} \in K_{e}, e=H, L$, can be written in the form (4).

According to Lemma 1, we can interchangeably use the probability measures $P_{H}$ and $P_{L}$ or the expected payoff functions $v_{H}($.$) and v_{L}($.$) of the plaintiff to describe the defendant's$ strategy. This result plays a critical role in the following analysis, where the geometric properties of $v_{H}$ and $v_{L}$ are extensively used.

\subsection{The plaintiff's strategy and the defendant's payoffs}

A plaintiff's behavioral strategy is represented by a map $\sigma:[a, b] \rightarrow[0,1]$, which specifies the probability $\sigma(x)$ that the plaintiff of type $x$ invests in case preparation. After observing the plaintiff's decision $e \in\{H, L\}$, the defendant revises her beliefs about the distribution of case strength. For a given plaintiff's strategy $\sigma$, we note $f_{e}$ and $F_{e}$, the density and c.d.f. of the defendant's posterior distributions. Assuming that both technologies are used in equilibrium, the revised densities are given by the Bayes' rule

$$
f_{H}(x)=\frac{\sigma(x) f(x)}{\int_{a}^{b} \sigma(t) f(t) d t} \quad \text { and } \quad f_{L}(x)=\frac{[1-\sigma(x)] f(x)}{\int_{a}^{b}[1-\sigma(t)] f(t) d t} .
$$

\footnotetext{
${ }^{16}$ More precisely, $v_{e}$ admits at every point a left- and a right-derivative, which coincide almost everywhere.
} 
Consequently, conditional on $e$, an offer $x$ yields the following expected revenues

$$
\begin{aligned}
\pi_{H}(x) & =\mu\left[-\left(x-\frac{T_{H}}{\mu}\right) F_{H}(x)-\int_{x}^{b} t f_{H}(t) d t\right]-t_{H}^{D} \\
\pi_{L}(x) & =-\left(x-T_{L}\right) F_{L}(x)-\int_{x}^{b} t f_{L}(t) d t-t_{L}^{D}
\end{aligned}
$$

to the defendant after $e=H$ or $e=L$. The difference between the above expressions of $\pi_{e}$ and the expression of $\pi_{\{e\}}$ used in the one-technology worlds of Section 2.2 is the underlying distributions of heterogeneity: the profits $\pi_{e}$ refer to the posterior distributions, while the prior distributions of case strength are used in $\pi_{\{e\}}$.

We cannot impose a priori that the investment probability $\sigma(x)$ is continuous in the case strength $x$. Indeed, continuity does not hold under complete information: the efficient configuration has $\sigma=0$ for low-types and $\sigma=1$ for high-types, thus an upward discontinuity at $\widetilde{x}$. To avoid useless complications, however, we assume that the function $\sigma$ has a left and a right limit at any point $x$, which are noted $\sigma\left(x^{-}\right)$and $\sigma\left(x^{+}\right)$respectively. ${ }^{17}$ It follows that the posterior densities $f_{H}$ and $f_{L}$ have left and right limits at any point $x$ and that the defendant's payoff function $\pi_{H}$ and $\pi_{L}$ have a left and a right derivative at any point $x$; the right derivatives are given by

$$
\pi_{H}^{\prime}\left(x^{+}\right) / \mu=-F_{H}(x)+\frac{T_{H}}{\mu} f_{H}\left(x^{+}\right) \text {and } \pi_{L}^{\prime}\left(x^{+}\right)=-F_{L}(x)+T_{L} f_{L}\left(x^{+}\right),
$$

the left derivatives are given by analog formulae. It is useful to observe that $\pi_{H}^{\prime}(x)$ and $\pi_{L}^{\prime}(x)$ are respectively equal, up to a positive multiplicative constant, to $\sigma(x) f(x)-\int_{a}^{x} \sigma \mathrm{d} F$ and $(1-\sigma(x)) f(x)-\int_{a}^{x}(1-\sigma) \mathrm{d} F$.

If the defendant randomizes between offers according to the probability distribution $P_{e}$, her payoff is $\Pi_{e}=\int_{a}^{b} \pi_{e}(x) \mathrm{d} P_{e}(x)$. According to Lemma 1, the defendant's strategy can also be expressed in terms of the utility she leaves to the plaintiff. In that case, her profits write as in Lemma 2.

Lemma 2. The conditional profits of the defendant given $e=H$ and $e=L$ can be expressed as functions of her strategy $\left(v_{H}, v_{L}\right)$ in the following way:

$$
\begin{aligned}
\Pi_{H} & =\int_{a}^{b}\left[-v_{H}(x)-\frac{T_{H}}{\mu} v_{H}^{\prime}(x)\right] f_{H}(x) \mathrm{d} x-c \\
\Pi_{L} & =\int_{a}^{b}\left[-v_{L}(x)-T_{L} v_{L}^{\prime}(x)\right] f_{L}(x) \mathrm{d} x .
\end{aligned}
$$

Proof. See Appendix B

\footnotetext{
${ }^{17}$ Mathematically, we impose the restriction that the plaintiff's strategy $\sigma$ belongs to the set of functions of bounded variation. The functions of bounded variation are differences between two nondecreasing functions. The set of discontinuity points of such a function can be infinite, but is necessarily countable. See W. P. Ziemer, Weakly Differentiable Functions: Sobolev Spaces and Functions of Bounded Variation, Graduate Texts in Mathematics. Springer-Verlag, New York, 1989.
} 
Finally, note that the unconditional defendant's profit, namely her expected profit before the investment decision is observed, is given by $\Pi_{\{H L\}}=\operatorname{Pr}(e=H) \Pi_{H}+\operatorname{Pr}(e=L) \Pi_{L}$, where $\operatorname{Pr}(e=H)=\int \sigma \mathrm{d} F$ and $\operatorname{Pr}(e=L)=\int(1-\sigma) \mathrm{d} F$.

\subsection{General properties of equilibria}

A perfect Bayesian equilibrium of the game is a function $\sigma^{*}($.$) and two probability measures$ $P_{H}^{*}$ and $P_{L}^{*}$ on $[a, b]$ such that (i) given $P_{H}^{*}$ and $P_{L}^{*}, \sigma^{*}(x)$ maximizes the expected payoff of type $x$, for all $x$ in $[a, b]$; (ii) given $\sigma^{*}, P_{e}^{*}$ maximizes the defendant expected payoff after she has observed $e$, for $e=H, L$; (iii) beliefs are updated according to Bayes' rule (8).

The support of a mixed strategy is the set of pure strategies to which a positive probability is assigned. For an offer to be in the support, a necessary condition is that it maximizes the defendant expected payoff. Whenever the maximum of her payoff is attained at many points, she is indifferent between the corresponding offers and can randomize between several of them. Formally:

$$
\operatorname{supp} P_{e}^{*} \subset \operatorname{argmax} \pi_{e},
$$

where $\pi_{e}$, given by (9) or (10), uses the updated beliefs. If, for $e=H$ or $L$, the defendant's payoff function attains its maximum at a unique point, then she makes the corresponding offer with probability 1: the support of distribution $P_{e}$ is thus a singleton, or, equivalently, $P_{e}$ is a mass point. (As seen above, this happens if all $x$ opt for the same technology.)

Lemma 3. Suppose that, in equilibrium, the investment probability $\operatorname{Pr}(e=H)$ is smaller than 1. Then, after observing $e=L$, the defendant makes no offer greater than $\widetilde{x}-t_{L}^{P}$. Formally: $v_{L}(\widetilde{x})=\widetilde{x}-t_{L}^{P}$.

Proof. We use a standard unraveling argument. Suppose that $v_{L}(\widetilde{x})>\widetilde{x}-t_{L}^{P}=\mu \widetilde{x}-t_{H}^{P}-c$. Since $v_{L}(b)=b-t_{L}^{P}<\mu b-t_{H}^{P}-c$, the curve $v_{L}$ must cross the segment $\mu x-t_{H}^{P}-c$ on $(\widetilde{x}, b]$. They only cross once since the segment has slope $\mu>1$ and $v_{L}$ has slope no greater than 1. Let $x_{0}$ be the unique intersection point. For $x>x_{0}$, we have: $v_{H}>v_{L}$, so the plaintiff chooses $e=H$ with probability $\sigma=1$. The defendant therefore knows that all the plaintiffs who choose $e=L$ necessarily have: $x \leq x_{0}$. Therefore she could reduce the utility $v_{L}($.$) by the constant amount z=v_{L}\left(x_{0}\right)-\left[x_{0}-t_{L}^{P}\right]>0$. Such a change would increase her payoff by $z \int_{a}^{x_{0}}(1-\sigma) \mathrm{d} F$, which is positive by assumption. We conclude that we must have: $v_{L}(\widetilde{x})=\widetilde{x}-t_{L}^{P}$, which is equivalent to saying that the support of $P_{L}$ is a subset of $[a, \widetilde{x}]$ or that the defendant, after observing $e=L$, does not make any offer greater than $\widetilde{x}-t_{L}^{P}$ with positive probability.

Corollary 1. In equilibrium, plaintiffs with strong cases invest in case preparation: $\sigma^{*}=1$ on $[\widetilde{x}, b]$.

Proof. The result, obviously, holds when the overall probability that $e=H, \int_{a}^{b} \sigma \mathrm{d} F$, is 1 . We concentrate, therefore, on the case where the overall probability of observing the basic technology, $\int_{a}^{b}(1-\sigma) \mathrm{d} F$, is positive. From Lemma 3 , for $x>\widetilde{x}$, we have: $v_{L}(x)=x-t_{L}^{P}<$ $\mu x-t_{H}^{P}-c$, so the plaintiff $x$ chooses $e=H, \sigma(x)=1$. 
According to Corollary 1, the investment decision of plaintiffs with strong cases is never distorted: both under symmetric and asymmetric information, high types efficiently invest in case preparation.

\section{Equilibria when trial costs are large}

This section is devoted to the case $\widetilde{x}<x_{H}^{*}$ or, equivalently, $\tau(\widetilde{x})<T_{H} / \mu$. This assumption expresses that, in the benchmark situation $\{H\}$, the marginal plaintiff $\widetilde{x}$ settles in equilibrium. The next proposition shows that, under this assumption, the only possible equilibrium configuration when both technologies are available is the same as in $\{H\}$.

Proposition 1. Assume $\widetilde{x}<x_{H}^{*}$. Then, in equilibrium, any plaintiff invests in case preparation: $\sigma^{*}=1$ on $[a, b]$. After observing $e=H$, the defendant offers $\mu x_{H}^{*}-t_{H}^{P}$ to settle the case.

Proof. First, we prove the existence of out-of-equilibrium beliefs that are consistent with this configuration. Suppose that, after she observes $e=L$, the defendant believes that the deviation comes from plaintiff $a$, and, accordingly, offers only $a-t_{L}^{P}$ to settle. It follows that a plaintiff of type $x$ receives utility $x-t_{L}^{P}$ if he does not invest, while he gets $v_{H}^{*}(x)=v_{\{H\}}\left(x ; x_{H}^{*}\right)>x-t_{L}^{P}$ if he invests (see Figure 3 ). In turn, any plaintiff invests and, as seen in Section 2.2, it is indeed optimal for the defendant to offer $\mu x_{H}^{*}-t_{H}^{P}$, leaving plaintiff $x_{H}^{*}$ indifferent between accepting or rejecting the offer.

Second, we check that the above configuration is the only possible one in equilibrium. The proof proceeds by contradiction. Assuming that the investment probability $\operatorname{Pr}(e=H)$ is smaller than 1 , we use the convexity of the functions $v_{H}$ and $v_{L}$ to show that there must exist $x_{1} \in(a, \widetilde{x}]$ such that, after observing $e=H$, the defendant makes the offer $\mu x_{1}-t_{H}^{P}$ with positive probability, and we show that this is not possible given the assumption $\widetilde{x}<x_{H}^{*}$. The detailed proof is presented in Appendix D.

According to Proposition 1, the plaintiff's strategy, as well as the defendant's strategy after she has observed $e=H$, are unique in equilibrium. Any distribution $P_{L}$ such that $v_{L} \leq v_{H}^{*}$ sustains the equilibrium. But the equilibrium configuration is unique, and is the same as in $\{H\}$. The uniqueness result is the heart of Proposition 1.

The intuition behind this result is fairly simple. When $T_{H} / \mu$ is large enough, the trial is relatively costly to at least one party and the plaintiff and/or the defendant are eager to settle. Therefore a relatively high settlement offer is made after $e=H$ which attracts all types of plaintiff.

The absence of the basic technology in equilibrium can harm some types of plaintiff. Indeed, assume that $x_{L}^{*}$ is larger than $x_{H}^{*}$ and such that $x_{L}^{*}-t_{L}^{P}$ is larger than $\mu x_{H}^{*}-t_{H}^{P}-c$ (more precisely, we are concerned by the case: $\widetilde{x}<x_{H}^{*}<x_{H}^{*}+(\mu-1)\left(x_{H}^{*}-\widetilde{x}\right)<x_{L}^{*}<b$ ). Then all plaintiffs who settle would prefer to do it for $x_{L}^{*}-t_{L}^{P}$ rather than $\mu x_{H}^{*}-t_{H}^{P}-c$. Yet the offer $x_{L}^{*}-t_{L}^{P}$ would only be made if all types selected $e=L$, which is not an equilibrium. 


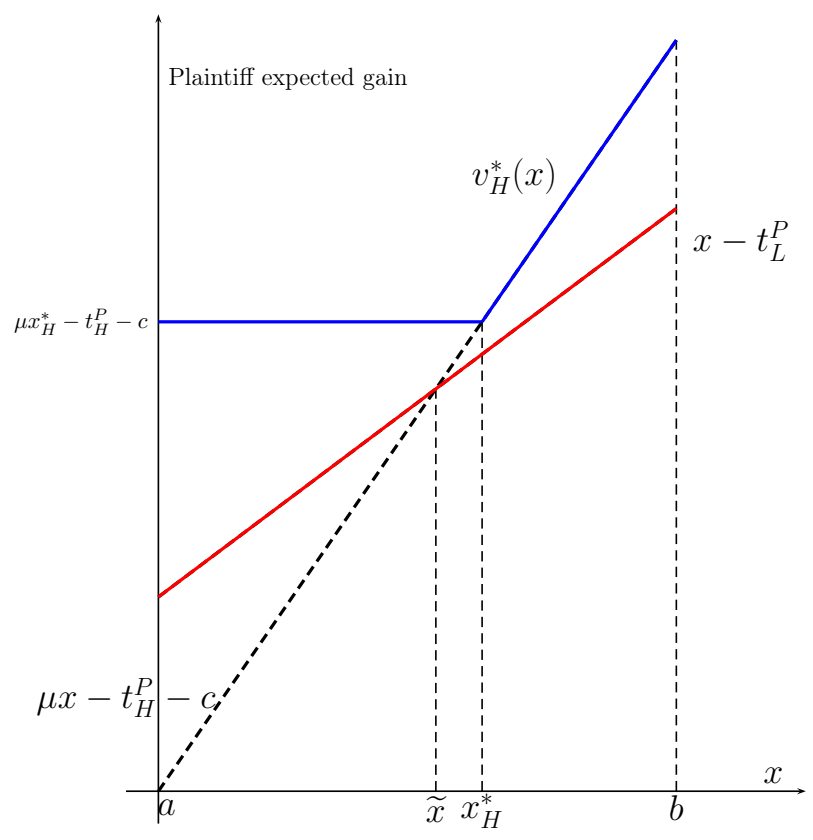

Figure 3: Equilibrium gains when $x_{H}^{*}>\widetilde{x}$

Finally, and more importantly, asymmetric information induces overinvestment. Plaintiffs with weak cases $(x<\widetilde{x})$ do not invest when information is symmetric, while they do when it is asymmetric. This choice is rewarding as they earn an informational rent. Part of the high-types (those who settle: $\widetilde{x}<x<x_{H}^{*}$ ) also benefit from asymmetric information.

\section{Equilibria when trial costs are low}

We now turn to the complementary case $x_{H}^{*}<\widetilde{x}$. Under this condition, assume that all types decide to invest in case preparation. Then the defendant makes the offer $\mu x_{H}^{*}-t_{H}^{P}$, which is rejected by all types above $x_{H}^{*}$. But the plaintiffs whose type lie between $x_{H}^{*}$ and $\widetilde{x}$ are better off, in court, with $e=L$ rather than with $e=H$ (see Figure 4). Therefore, it can no longer be an equilibrium for all types of plaintiff to invest.

We know from Corollary 1 that high types invest in case preparation. The following proposition goes a step further towards the characterization of the equilibrium.

Proposition 2. Assume that $x_{H}^{*}<\widetilde{x}$. In equilibrium, plaintiffs with strong cases $(x>\widetilde{x})$ invest and go to court; plaintiffs with weak cases $(x \leq \widetilde{x})$ are indifferent between investing or not. Formally: $v_{H}=v_{L}$ on $[a, \widetilde{x}]$.

Proof. Since the probability of investment is smaller than one, we know from Lemma 3 that $v_{L}(\widetilde{x})=\widetilde{x}-t_{L}^{P}$. In Appendix E, we show, by using the convexity of the functions $v_{H}$ and $v_{L}$, that the defendant, after observing $e=H$, makes no offer greater than $\mu \widetilde{x}-t_{H}^{P}$, which 


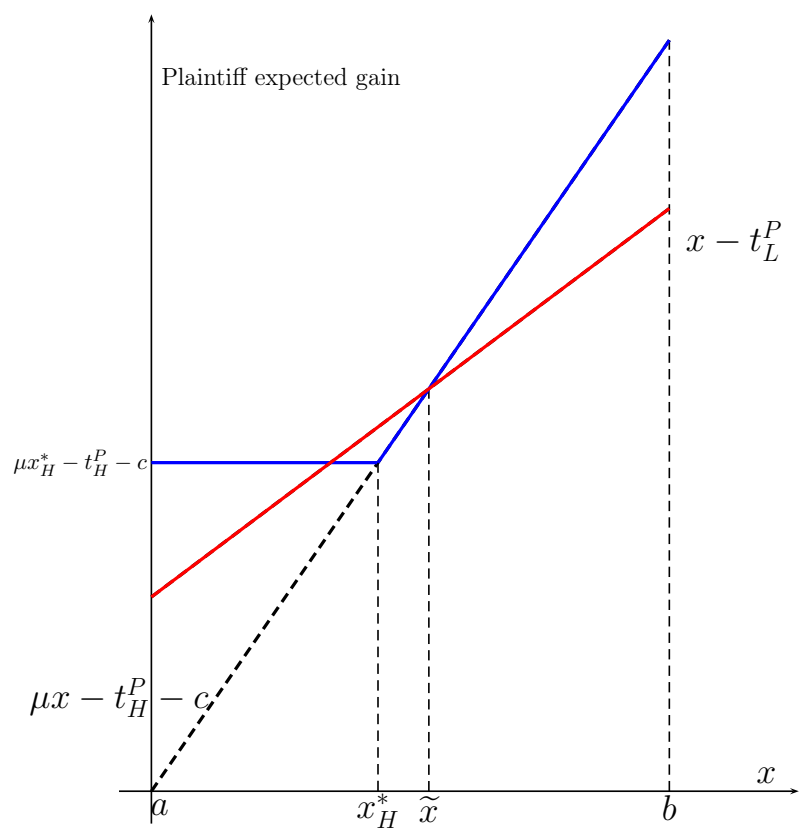

Figure 4: When $x_{H}^{*}<\widetilde{x}$, all types choosing $e=H$ is no longer an equilibrium

is equivalent to $v_{H}(\widetilde{x})=v_{L}(\widetilde{x})$. It follows that $v_{H}(x)=\mu x-t_{H}^{P}-c>v_{L}(x)=x-t_{L}^{P}$ for $x>\widetilde{x}$, and that plaintiffs with strong cases invest and go to court.

We now turn to plaintiffs with weak cases, and show that $v_{L}=v_{H}$ on $[a, \widetilde{x}]$. We proceed by contradiction. Suppose that there exists $x<\widetilde{x}$ such that $v_{L}(x) \neq v_{H}(x)$, say, for instance, $v_{L}(x)<v_{H}(x)$. Since $v_{L}=v_{H}$ at $\widetilde{x}$, there exists $x_{1}$, with $x<x_{1} \leq \widetilde{x}$ such that $v_{L}=v_{H}$ at $x_{1}$ and $v_{L}<v_{H}$ on $\left[x, x_{1}\right)$. We have $v_{L}<v_{H}, \sigma=1$ and $f_{L}=0$ on $\left[x, x_{1}\right)$.

Suppose first that $F_{L}(x)$ is positive. In this case, we have: $\pi_{L}^{\prime}=-F_{L}+T_{L} f_{L}=-F_{L}<0$ on $\left[x, x_{1}\right)$, so $\pi_{L}$ does not attain its maximum in this interval, which, therefore, does not intersect the support of $P_{L}$. Applying Lemma C.2, we conclude that $v_{L}$ is affine on $\left[x, x_{1}\right)$. More generally, the argument shows that $v_{L}$ is affine as long as it is below $v_{H}$. Since $v_{H}$ is convex and $v_{H}\left(x_{1}\right)=v_{L}\left(x_{1}\right)$, the only possibility is that $v_{L}<v_{H}$ and $\sigma=1$, on the whole interval $\left[a, x_{1}\right]$, which contradicts $F_{L}(x)>0$.

It follows that $F_{L}(x)=0$. Applying Lemma C.1, we conclude that $v_{L}$ is constant on $[a, x]$. But this, again, is impossible as $v_{H}\left(x_{1}\right)=v_{L}\left(x_{1}\right), v_{H}>v_{L}$ on a left neighborhood of $x_{1}$, and $v_{H}$ is nondecreasing.

It follows that $v_{H} \leq v_{L}$ on $[a, \widetilde{x}]$. The proof of $v_{L} \leq v_{H}$ is symmetric.

The following proposition characterizes the defendant's strategy in equilibrium.

Proposition 3. Assume that $x_{H}^{*}<\widetilde{x}$. After $e=L$, the defendant makes a single offer, $\widehat{x}-t_{L}^{P}$, where $\widehat{x}$ lies between $x_{H}^{*}$ and $x_{L}^{*}$ and $\widehat{x}<\widetilde{x}$; after $e=H$, she offers $\mu \widehat{x}-t_{H}^{P}$ with 
probability $1 / \mu$ and $\mu \widetilde{x}-t_{H}^{P}$ with probability $1-1 / \mu$.

Proof. See Appendix F

The key point in Proposition 3 is that the support of $P_{L}$ must be a singleton. If the plaintiff has not invested, the defendant does not randomize. ${ }^{18}$ In the other case, she randomizes between exactly two offers. Formally, if $P_{L}$ is the singleton $\{\widehat{x}\}$, then the support of $P_{H}$ must be the pair $\{\widehat{x}, \widetilde{x}\}$. The weights of $\widehat{x}$ and $\widetilde{x}$ are $1 / \mu$ and $1-1 / \mu$ respectively. The equilibrium settlement strategy of the plaintiff immediately follows Proposition 3.

Corollary 2 (Settlement and trial conditional on plaintiff's type and investment decision). Assume that $x_{H}^{*}<\widetilde{x}$ and consider a plaintiff of type $x$. After case preparation, he settles out of court if $x \leq \widehat{x}$, declines the low offer and accepts the high one if $\widehat{x}<x \leq \widetilde{x}$, goes to court otherwise. If the plaintiff has not invested, he goes to court if and only if $x>\widehat{x}$.

We now examine the behavior of plaintiffs with weak cases $(x<\widetilde{x})$. The main result is that the fraction of low types investing in the case preparation is positive and constant across equilibria. This contrasts with the symmetric information case, where low plaintiffs never invest in case preparation. These weak case types who overinvest in equilibrium are certain to regret their choice if they cannot settle and end in court. As for them their expected net gain at trial is larger after $e=L$ rather than after $e=H$.

Corollary 3 (Overinvestment by plaintiffs with weak cases). The fraction of plaintiffs with weak cases $(x<\widetilde{x})$ who invest in case preparation is given by

$$
\operatorname{Pr}(e=H \mid x<\widetilde{x})=\frac{T_{H}}{\mu} \frac{f(\widetilde{x})}{F(\widetilde{x})}
$$

Proof. Since $\widetilde{x}$ belongs to the support of $P_{H}$, Lemma C.3 implies that $\sigma\left(\widetilde{x}^{+}\right) \leq \sigma\left(\widetilde{x}^{-}\right)$. As $\sigma\left(\widetilde{x}^{+}\right)=1$ it means that $\sigma($.$) is continuous at \widetilde{x}$, with $\sigma(\widetilde{x})=1$. Therefore, the function $\pi_{H}$ is differentiable at $\widetilde{x}$; since $\pi_{H}$ is maximal at $\widetilde{x}$, we have $\pi_{H}^{\prime}(\widetilde{x})=0$, which writes:

$$
F_{H}(\widetilde{x})=\frac{T_{H}}{\mu} f_{H}(\widetilde{x})=\frac{T_{H}}{\mu} \frac{f(\widetilde{x})}{\operatorname{Pr}(e=H)},
$$

which, combined with

$$
\operatorname{Pr}(e=H \mid x<\widetilde{x})=\frac{1}{F(\widetilde{x})} \int_{a}^{\widetilde{x}} \sigma(x) f(x) \mathrm{d} x=\frac{1}{F(\widetilde{x})} \operatorname{Pr}(e=H) F_{H}(\widetilde{x}),
$$

yields (11).

The next corollary shows the plaintiff's gain in equilibrium, denoted $v^{*}(x)$, which is represented on Figure 5.

\footnotetext{
${ }^{18}$ This property follows from the log-concavity of the distribution of types, see section F.3.
} 


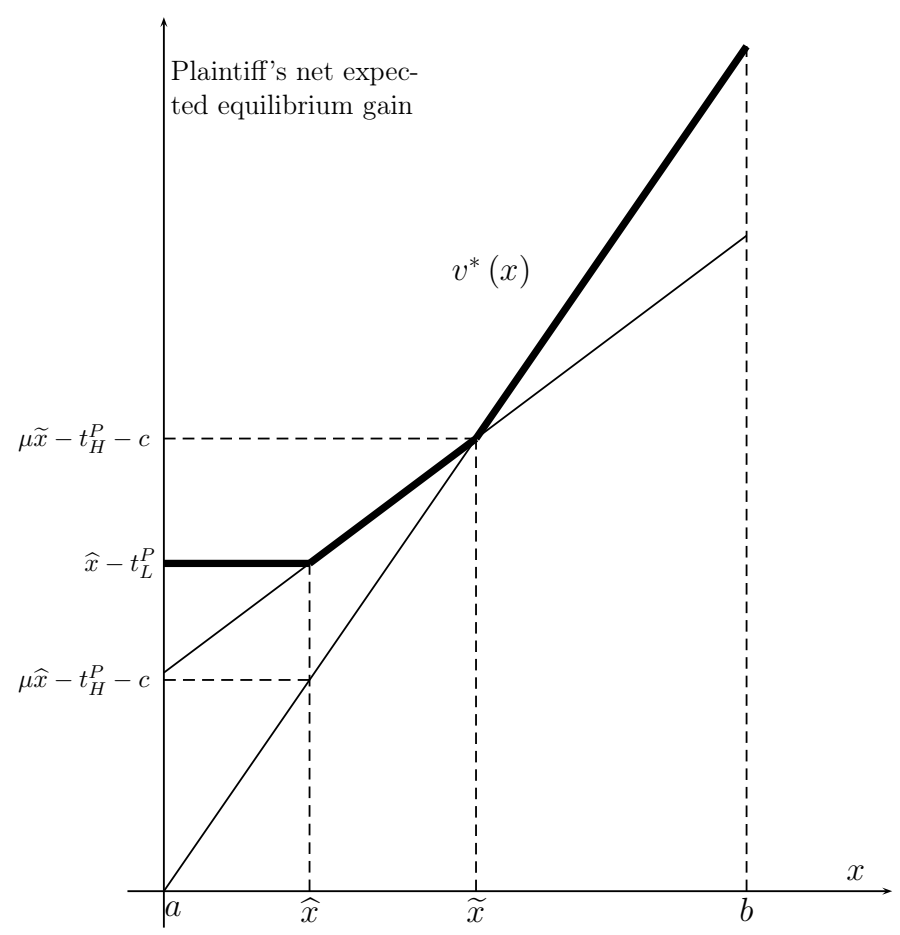

Figure 5: Equilibrium payoff of the plaintiff and offers of the defendant

Corollary 4 (Plaintiff's payoff). Assume that $x_{H}^{*}<\widetilde{x}$. In equilibrium, the net expected payoff of the plaintiff of type $x$ is $\widehat{x}-t_{L}^{P}$ if $a \leq x \leq \widehat{x}, x-t_{L}^{P}$ if $\widehat{x} \leq x \leq \widetilde{x}$, and $\mu x-t_{H}^{P}-c$ if $\widetilde{x} \leq x \leq b$.

Plaintiffs with very weak cases $(x<\widehat{x})$ never go to court, and earn an informational rent compared to the symmetric information case (see Figure 2). In contrast, types above $\widehat{x}$ have the same expected payoff under symmetric and asymmetric information. Plaintiffs with intermediate cases $(\widehat{x} \leq x \leq \widetilde{x})$ go to court with probability one if they do not invest, with probability $1 / \mu$ if they do. Plaintiffs with strong cases invest and go to court.

Note, that in Hay (1995)'s model both the plaintiff and the defendant have (on average) the same payoff whether or not the effort is hidden. The lack of settlement entails no loss (despite the parties' costs of trial) for each party: the trial costs of the defendant (resp. plaintiff) are exactly compensated by the fact that with some probability a lower offer is accepted (resp. a high offer is made while no effort has been undertook). In our model, incomplete information entails a social cost and we discuss the welfare impact of the availability of different case preparation strategies.

Next, we examine the defendant's unconditional payoff in equilibrium, that is, her profit before she observes the plaintiff's investment decision. A first way to compute her profit is 
to consider her response to each plaintiff type's decision. We get:

$$
\begin{aligned}
-\Pi_{\{H L\}}^{*} & =\int_{a}^{\widehat{x}}\left\{\sigma\left[\frac{1}{\mu}\left(\mu \widehat{x}-t_{H}^{P}\right)+(1-1 / \mu)\left(\mu \widetilde{x}-t_{H}^{P}\right)\right]+(1-\sigma)\left(\widehat{x}-t_{L}^{P}\right)\right\} f(x) \mathrm{d} x \\
& +\int_{\widehat{x}}^{\widetilde{x}}\left\{\sigma\left[\frac{1}{\mu}\left(\mu x+t_{H}^{D}\right)+(1-1 / \mu)\left(\mu \widetilde{x}-t_{H}^{P}\right)\right]+(1-\sigma)\left(x+t_{L}^{D}\right)\right\} f(x) \mathrm{d} x \\
& +\int_{\widetilde{x}}^{b}\left[\mu x+t_{H}^{D}\right] f(x) \mathrm{d} x .
\end{aligned}
$$

The first line correspond to plaintiffs with very weak cases $(x<\widehat{x})$, who invest with probability $\sigma(x)$. If the defendant observes that the plaintiff has not invested, she offers $\widehat{x}-t_{L}^{P}$, and the plaintiff agrees to settle; if she observes that he has invested, she offers $\mu \widehat{x}-t_{H}^{P}$ with probability $1 / \mu$ and $\mu \widetilde{x}-t_{H}^{P}$ with probability $1-1 / \mu$. Again, the plaintiff accepts both offers. The second and third lines can be interpreted in the same fashion. In Appendix G, we derive another expression of the defendant's payoff, which will prove useful below.

Corollary 5 (Defendant's payoff). Assume that $x_{H}^{*}<\widetilde{x}$. In equilibrium, the net expected payoff of the defendant is given by

$$
\begin{aligned}
-\Pi_{\{H L\}}^{*}= & \left(\widehat{x}-t_{L}^{P}\right) \int_{a}^{\widehat{x}} f(x) \mathrm{d} x+\int_{\widehat{x}}^{\widetilde{x}}\left[x+t_{L}^{D}\right] f(x) \mathrm{d} x+\int_{\widetilde{x}}^{b}\left[\mu x+t_{H}^{D}\right] f(x) \mathrm{d} x \\
& +c \frac{T_{H}}{\mu} f(\widetilde{x})+\left(\frac{T_{H}}{\mu}-T_{L}\right) \int_{\widehat{x}}^{\widetilde{x}} \sigma(x) f(x) \mathrm{d} x .
\end{aligned}
$$

The last term of the first line of (12) comes from the plaintiffs with strong cases $(x \geq \widetilde{x})$, who invest and go to court. The first and second terms of the first line are the amounts that the defendant would pay absent investment by low plaintiffs $(x<\widetilde{x})$. The first term accounts for the types between $a$ and $\widehat{x}$ who settle and the second for the types between $\widehat{x}$ and $\widetilde{x}$ who do not. Yet in equilibrium, a fraction of plaintiffs with weak cases do invest, which is reflected by the two correction terms of the second line. First, investment by low plaintiffs entails an extra cost $c \operatorname{Pr}(e=H$ and $x<\widetilde{x})$, which is fully "passed on" to the defendant (since low plaintiffs must be kept indifferent between investing or not). Second, for types between $\widehat{x}$ and $\widetilde{x}$, investment alters the expected trial costs, which explains the second term of the second line of (12).

Proposition 3 does not characterize the plaintiff's strategy $\sigma($.$) ; in particular, it does$ not rule out the possibility of multiple equilibria associated with different functions $\sigma$. We now exhibit a fully specified equilibrium $\left(\sigma^{*}, P_{H}, P_{L}\right)$.

Proposition 4. Assume that $x_{H}^{*}<\widetilde{x}$. If $T_{L}=T_{H} / \mu$, we set $\widehat{x}=x_{L}^{*}=x_{H}^{*}$, otherwise we define $\widehat{x}$ as the highest root to the equation

$$
\frac{f(\widetilde{x})}{f(x)} \exp \left[\frac{\mu}{T_{H}}\left(x_{H}^{*}-x\right)\right]=\frac{\tau(x)-T_{L}}{T_{H} / \mu-T_{L}} .
$$


We define the defendant's strategy $\left(P_{H}, P_{L}\right)$ as in Proposition 3. We define the plaintiff's strategy by

$$
\sigma^{*}(x)= \begin{cases}\left(f(\widetilde{x}) / f\left(x_{H}^{*}\right)\right) \exp \left[\frac{\mu}{T_{H}}\left(x_{H}^{*}-\widetilde{x}\right)\right] & \text { for } a \leq x \leq x_{H}^{*} \\ (f(\widetilde{x}) / f(x)) \exp \left[\frac{\mu}{T_{H}}(x-\widetilde{x})\right] & \text { for } x_{H}^{*} \leq x \leq \widetilde{x} \\ 1 & \text { for } \widetilde{x} \leq x \leq b\end{cases}
$$

if $T_{L} \geq T_{H} / \mu$, and

$$
\sigma^{*}(x)= \begin{cases}1-(1-\sigma(\widehat{x}))\left(f(\widehat{x}) / f\left(x_{L}^{*}\right)\right) \exp \left[\frac{1}{T_{L}}\left(x_{L}^{*}-\widehat{x}\right)\right] & \text { for } a \leq x \leq x_{L}^{*} \\ 1-(1-\sigma(\widehat{x}))(f(\widehat{x}) / f(x)) \exp \left[\frac{1}{T_{L}}(x-\widehat{x})\right] & \text { for } x_{L}^{*} \leq x \leq \widehat{x} \\ (f(\widetilde{x}) / f(x)) \exp \left[\frac{\mu}{T_{H}}(x-\widetilde{x})\right] & \text { for } \widehat{x} \leq x \leq \widetilde{x}, \\ 1 & \text { for } \widetilde{x} \leq x \leq b\end{cases}
$$

if $T_{L}<T_{H} / \mu$. Then $\left(\sigma^{*}, P_{H}, P_{L}\right)$ is an equilibrium.

The equilibrium of Proposition 4 is represented on Figures 6a and 6b. The formal check that the given strategies form an equilibrium is relegated in Appendix $\mathrm{H}$.

The logic behind the shape of $\sigma^{*}($.$) is the following. As stated in Proposition 3, the$ defendant makes a unique offer, $\widehat{x}$, after $e=L$, and she randomizes between $\widehat{x}$ and $\widetilde{x}$ after $e=H$. Accordingly, $\pi_{L}$ has to attain its maximum at $x=\widehat{x}$, and $\pi_{H}$ at both $\widehat{x}$ and $\widetilde{x}$. The function $\sigma^{*}$ of Proposition 4 is such that $\pi_{H}$ is constant between $\widehat{x}$ and $\widetilde{x}$. In Appendix $\mathrm{H}$, we check that $\pi_{L}$ is maximal at $\widehat{x}$ on the interval $[\widehat{x}, b]$. On $[a, \widehat{x}], \sigma^{*}($.$) is such that \pi_{H}$ and $\pi_{L}$ are nondecreasing and such that enough low types choose to invest, in accordance with Equation (11). When $T_{L} \geq T_{H} / \mu$ (Figure 6a), this can be achieved by maintaining $\pi_{H}$ flat between $x_{H}^{*}$ and $\widehat{x}$, and keeping $\sigma^{*}($.$) constant between a$ and $x_{H}^{*}$. When $T_{L}>T_{H} / \mu$ (Figure $6 \mathrm{~b}$ ), this choice of $\sigma^{*}$ below $\widehat{x}$ is not consistent with the equilibrium requirements, as it would not give enough weight to $e=H$ and Equation (11) would be violated. As a consequence, $\sigma^{*}($.$) has to decrease between a$ and $\widehat{x}$. A way to satisfy Equation (11) is to choose $\sigma^{*}($.$) between x_{L}^{*}$ and $\widehat{x}$ such that $\pi_{L}$ is flat and then to maintain $\sigma^{*}($.$) constant$ between $a$ and $x_{L}^{*}$. In Appendix $\mathrm{H}$, it is checked that, in these circumstances, $\pi_{H}$ is increasing on $[a, \widehat{x}]$.

In the equilibrium of Proposition 4, all plaintiffs with weak cases $(x<\widetilde{x})$ resort to a mixed strategy. Obviously, one can construct equilibria where many low types play in pure strategy, provided that the probability of investment conditional on $x<\widetilde{x}$, given (11), is not affected. However, mixed strategies cannot not be entirely ruled out, as shown in the following remark (proved in Appendix F.4).

Remark 1. The mixed strategy of the plaintiff $\widehat{x}$ must be non degenerate: $0<\sigma^{*}(\widehat{x})<1$. 

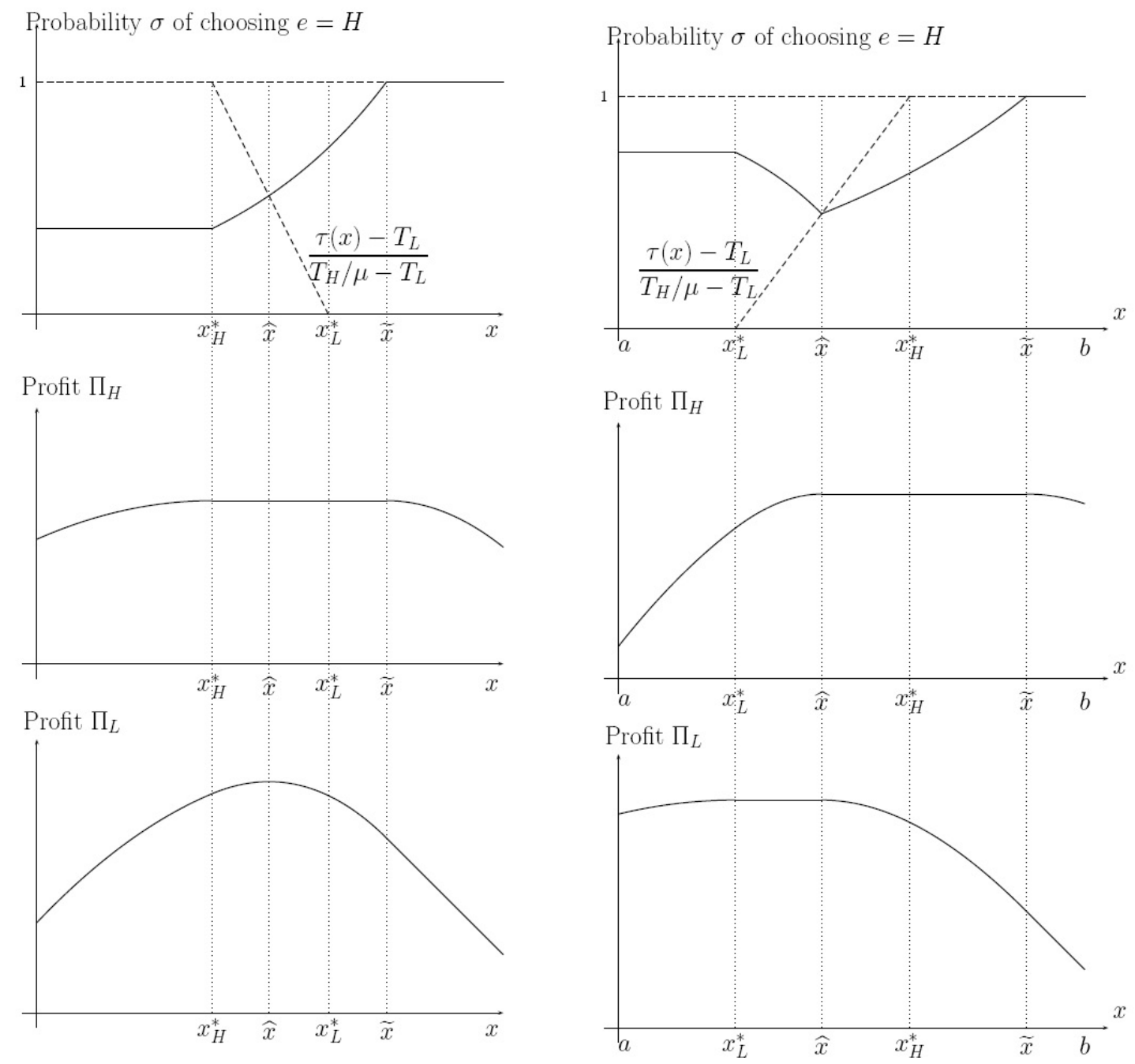

Figure 6a: $x_{H}^{*}<\widetilde{x}$ and $x_{H}^{*}<x_{L}^{*}$

Figure 6b: $x_{L}^{*}<x_{H}^{*}<\widetilde{x}$

Parameter values: $F$ uniform on $[1,3], t_{H}^{D}=t_{L}^{D}=$ $0, t_{H}^{P}=1.5, t_{L}^{P}=1.25, c=2.25$, and $\mu=2$.

Parameter values: $F$ uniform on $[1,3], t_{H}^{D}=t_{L}^{D}=$ $0, t_{H}^{P}=2.5, t_{L}^{P}=0.5, c=0.75$, and $\mu=2$.

As $\widehat{x}$ belongs to the supports of both $P_{L}$ and $P_{H}, \sigma^{*}$ is continuous at $\widehat{x}$ (Lemma C.3), Remark 1 implies that a positive mass of plaintiffs plays in mixed strategy. ${ }^{19}$

\section{Discussion}

We now present qualitative properties of the equilibria and comparative statics results that illustrate the strategic effects at work. First, we show how the introduction of a publicly ob-

\footnotetext{
${ }^{19}$ Admittedly, these results partly follow from our assumption that $\sigma$ is a function of bounded variations (see footnote 17). Recall, however, that this restriction allows for an infinite number of discontinuity points.
} 
servable investment decision by the plaintiff alters Bebchuk's equilibrium pattern. Second, we examine the extent of overinvestment by plaintiffs with weak cases, and explain how it varies with the primitives of the model. Third, we study the welfare effects of introducing a new technology, starting from a one-technology world.

\subsection{The trial probability may locally decrease with case strength}

Figures $7 \mathrm{a}$ and $7 \mathrm{~b}$ plot the probabilities of trial conditional on the investment choice as functions of the case strength $x$. As stated in Lemma 1, these probabilities are nondecreasing in $x$. A plaintiff who expects large damages in court is less likely to settle. Yet Figure 7c shows that this property does not extend to the unconditional trial probability: a plaintiff with a stronger case can settle more often than a plaintiff with a weaker case.

Corollary 6. Assume that $x_{H}^{*}<\widetilde{x}$. At the equilibrium of Proposition 4, the unconditional trial probability decreases with case strength on $[\widehat{x}, \widetilde{x}]$.

This result is in sharp contrast with Bebchuk (1984), as well as with Reinganum and Wilde (1986), where the probability of settlement is decreasing in $x$. In the present model, the endogenous investment decision entails a selection effect, which can delete monotonicity, as shown on Figure 7c. For a given investment decision, the trial probability increases with case strength. Ex ante, however, the opposite result may hold as plaintiffs with stronger cases are more likely to invest in case preparation.

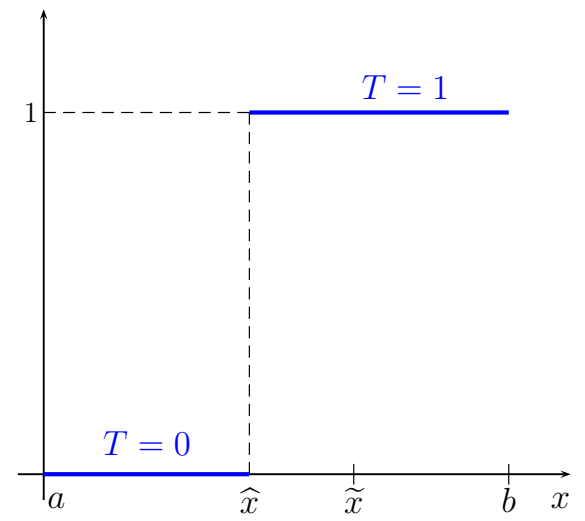

Figure 7a: Proba. of trial after $e=L$

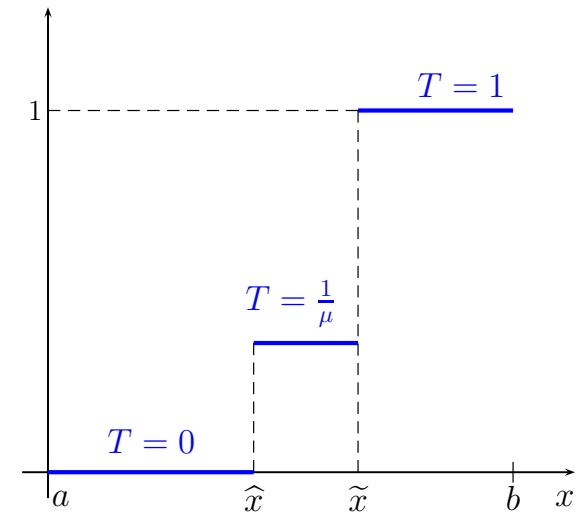

Figure 7b: Proba. of trial after $e=H$

\subsection{The extent of overinvestment}

Plaintiffs with weak cases $(x<\widetilde{x})$ who decide to invest in case preparation can be called bluffers. If the defendant calls their bluff and brings them to court, they regret their choice. When $\widetilde{x}<x_{H}^{*}$, the proportion of bluffers is one, as all types invest, and the bluff is always successful as the defendant settles with all types below $x_{H}^{*}>\widetilde{x}$. When $x_{H}^{*}<\widetilde{x}$, however, this proportion, given by (11), is lower than one; moreover, the bluff is successful with 


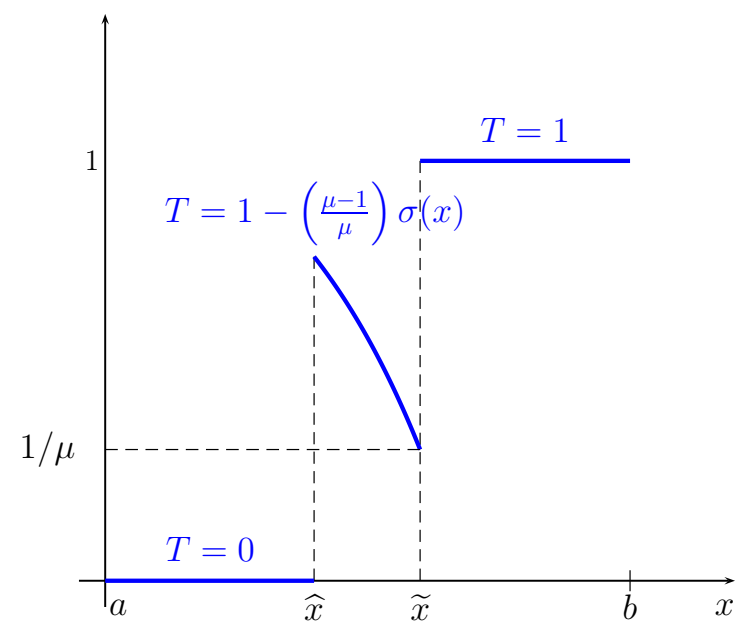

Figure 7c: Unconditional probability of trial

probability $1-1 / \mu$ only as the defendant randomizes between a low and a high offer after observing that the plaintiff has invested. The following Lemma describes how the extent of bluff varies with the primitives of the model.

Lemma 4 (Proportion of bluffers). In equilibrium, the proportion of bluffers becomes larger when (other things being equal):

i) The defendant's trial cost, $t_{H}^{D}$, increases.

ii) The plaintiff's trial cost after $e=L, t_{L}^{P}$, increases.

iii) The sunk cost of investment, $c$, decreases.

Proof. i) An increase of $t_{H}^{D}$ increases $T_{H}$, but not does not change $\widetilde{x}$. Moreover, the proportion of bluffers tends to one as $t_{H}^{D}$ goes to $\mu \tau(\widetilde{x})-t_{H}^{P}$, all other parameters being fixed. ii) and iii) If $t_{L}^{P}$ increases (resp. $c$ decreases), $T_{H} / \mu$ is not affected, while $\widetilde{x}$ decreases, therefore $\frac{T_{H}}{\mu} / \tau(\widetilde{x})$ increases.

\subsection{The welfare effects of introducing a second technology}

At the end of Section 2, we compared the expected litigation costs $\mathcal{C}_{\{H\}}^{*}$ and $\mathcal{C}_{\{L\}}^{*}$ in the two benchmark situations. We now investigate the welfare effects of introducing the costly technology, $H$, when only the basic one, $L$, is available, and, symmetrically, of introducing $L$ when only $H$ is available. When $x_{H}^{*} \geq \widetilde{x}$, the effect is obvious as the equilibrium configuration is the same in $\{H\}$ and in $\{H L\}$. Hereafter, we focus on the case $x_{H}^{*}<\widetilde{x}$. The total litigation costs in $\{H L\}$ are given by:

$$
\mathcal{C}_{\{H L\}}^{*}=\int_{\widehat{x}}^{\widetilde{x}}\left[\sigma(x) \frac{T_{H}}{\mu}+(1-\sigma(x)) T_{L}\right] f(x) \mathrm{d} x+[1-F(\widetilde{x})] T_{H}+c \operatorname{Pr}(e=H) .
$$

Plaintiffs with very weak cases $(x<\widehat{x})$ settle. Plaintiffs with intermediate cases $(\widehat{x} \leq x \leq \widetilde{x})$ invest with probability $\sigma$, then go to court with probability $1 / \mu$, or do not invest (probability 
$1-\sigma)$ and go to court with certainty; their contribution to the total costs is therefore $\sigma(x) \frac{T_{H}}{\mu}+(1-\sigma(x)) T_{L}$. Finally, plaintiffs with strong cases $(x>\widetilde{x})$ invest and go to court, generating trial costs $T_{H}$.

Starting from a one-technology world $\{e\}$, the introduction of the case preparation technology has four effects on total costs. First, the critical case strength below which plaintiffs always settle is $x_{e}^{*}$ in $\{e\}$ and $\widehat{x}$ in $\{H L\}$. The threshold $\widehat{x}$ is lower or higher than $x_{e}^{*}$ depending on the ordering of $T_{L}$ and $T_{H} / \mu$. Second, in a (possibly empty) intermediate region $\left[\max \left(x_{e}^{*}, \widehat{x}\right), \widetilde{x}\right], T_{e}$ is replaced by $\sigma(x) \frac{T_{H}}{\mu}+(1-\sigma(x)) T_{L}$, which is certainly smaller than $T_{H}$, and may be lower or higher than $T_{L}$ depending, again, on the ordering of $T_{L}$ and $T_{H} / \mu$. Third, the introduction of $L$ starting from $\{H\}$ does not change the contribution of plaintiffs with strong cases; the introduction of $H$ starting from $\{L\}$ increases their contribution from $T_{L}$ to $T_{H}$. Fourth, the introduction of a second technology modifies the overall investment probability $\operatorname{Pr}(e=H)$, and, in turn, the weight of the sunk cost $c$.

The overall effect is ambiguous in general, but can be determined in the particular case where the case preparation investment multiplies the total trial cost by the same factor as the expected award in court: $T_{H} / \mu=T_{L}$. Under this assumption, the trial probability is the same in $\{H\}$ and in $\{L\}$, so the direct cost effect implies $\mathcal{C}_{\{H\}}^{*}>\mathcal{C}_{\{L\}}^{*}$. Furthermore, in $\{H L\}$, plaintiffs with weak cases generate the same expected trial costs, whether they invest $\left(T_{H} / \mu\right)$ or not $\left(T_{L}\right)$.

Proposition 5 (Pure bluff effect). Assume that $x_{H}^{*}=x_{L}^{*}<\widetilde{x}$. Then

i) From $\{L\}$, the introduction of $H$ reduces the trial probability, raises the expected litigation costs, benefits the plaintiff, irrespective of his type, and harms the defendant.

ii) From $\{H\}$, the introduction of $L$ reduces the trial probability and the expected litigation costs, is beneficial to the plaintiff as well as, if $x_{L}^{*} \geq T_{L}$, to the defendant.

Proof. If $x_{H}^{*}=x_{L}^{*}$, we have, by Proposition 3: $\widehat{x}=x_{H}^{*}=x_{L}^{*}$. In the two benchmark situations, plaintiffs settle if and only if their type is below $\widehat{x}$, and the probability of settlement is $F\left(x_{H}^{*}\right)=F\left(x_{L}^{*}\right)=F(\widehat{x})$. When both technologies are available, plaintiffs with type $x \leq \widehat{x}$ continue to settle; but plaintiffs with type $x \in[\widehat{x}, \widehat{x}]$ now settle when they invest and receive the high offer $\mu \widetilde{x}-t_{H}^{P}$, which occurs with probability $\sigma(x) \cdot[1-1 / \mu]$. The probability of settlement is, therefore, increased by $(1-1 / \mu) \int_{\widehat{x}}^{\widetilde{x}} \sigma(x) f(x) \mathrm{d} x$, which is positive, since $\sigma(\widetilde{x})=1$ and $\sigma$ is continuous at $\widetilde{x}$ (see Appendix $\mathrm{F}$ ).

Under the assumptions of the Proposition, the total costs simplify into $\mathcal{C}_{\{H L\}}^{*}=c \operatorname{Pr}(e=$ $H)+[F(\widetilde{x})-F(\widehat{x})] T_{L}+[1-F(\widetilde{x})] T_{H}$. Comparing with (3) yields $\mathcal{C}_{\{L\}}^{*}<\mathcal{C}_{\{H L\}}^{*}<\mathcal{C}_{\{H\}}^{*}$.

It is straightforward to check that the plaintiff's payoff in $\{H L\}$, which is represented on Figure 5, is uniformly greater than his payoffs $v_{\{L\}}(x ; \widehat{x})$ and $v_{\{H\}}(x ; \widehat{x})$ in the onetechnology worlds. It follows that the plaintiff, whatever his type, prefers $\{H L\}$ to both $\{H\}$ and $\{L\}$.

Starting from $\{L\}$, the introduction of the costly technology reduces total welfare and benefits the plaintiff (irrespective of his type); it must therefore harm the defendant. Starting from $\{H\}$, the introduction of the basic technology raises total welfare; so it might 
benefit both parties. Item ii of the Proposition, proved in Appendix I, establishes that it is indeed the case under the additional condition $x_{L}^{*} \geq T_{L}$.

The results of Proposition 5 are driven by a pure bluff effect. Under the assumption $x_{H}^{*}=x_{L}^{*}<\widetilde{x}$, the incentives to settle in $\{H\}$ and in $\{L\}$ are identical; the lower threshold for settlement, $\widehat{x}$, coincide with $x_{H}^{*}=x_{L}^{*}$. Yet a fraction of types above this threshold invests in the costly technology, and is rewarded by a generous settlement offer, thereby reducing the overall probability of trial.

Part ii of Proposition 5 shows that, if $T_{L} \leq x_{L}^{*}=x_{H}^{*}<\widetilde{x}$, the introduction of the basic technology, starting from the situation $\{H\}$, is Pareto-improving. The left inequality holds, for instance, when the distribution of case strength is uniform as $x_{L}^{*}=a+T_{L}$.

Under the assumptions of Proposition 5, the equilibrium total cost when both technologies are available lies between $\mathcal{C}_{\{H\}}^{*}$ and $\mathcal{C}_{\{L\}}^{*}$. The final proposition shows that this is not true in general. More interestingly, while overinvestment generates inefficient sunk costs, it may also trigger more settlements through the bluff effect, thereby reducing trial costs. The overall effect may be a reduction of the litigation costs.

Proposition 6. Starting from $\{L\}$, the introduction of $H$ may reduce the expected litigation costs, even when $H$ alone leads to higher costs than $L$ alone. In other words, the ordering $\mathcal{C}_{\{H L\}}^{*}<\mathcal{C}_{\{L\}}^{*}<\mathcal{C}_{\{H\}}^{*}$ is possible.

To construct an example, we choose $\widetilde{x}$ close to $b$, so as to minimize both the preparation and the trial costs generated by plaintiffs with strong cases. We choose $x_{L}^{*}<x_{H}^{*}$, so that trial occurs less often in $\{H\}$ than in $\{L\}$. But we make sure that, in the comparison of $\{H\}$ and $\{L\}$, the strategic effect does not offset the cost effect, so that $\mathcal{C}_{\{L\}}^{*}<\mathcal{C}_{\{H\}}^{*}$. Next, we compare $\{L\}$ and $\{H L\}$. First the sunk preparation cost tends to make $\mathcal{C}_{\{H L\}}^{*}$ higher than $\mathcal{C}_{\{L\}}^{*}$. Second, $\sigma T_{H} / \mu+(1-\sigma) T_{L}$ is larger than $T_{L}$, which pushes in the same direction. On the other hand, because of $\widehat{x}>x_{L}^{*}$, more plaintiffs settle in $\{H L\}$ than in $\{L\}$, which tends to make $\mathcal{C}_{\{H L\}}^{*}$ lower than $\mathcal{C}_{\{L\}}^{*}$. We now exhibit a set of parameters such that the latter effect dominates, which proves Proposition 6.

We assume that $x$ uniformly distributed on [1,3], and set: $c=0.08, t_{L}^{P}=0.5, t_{L}^{D}=1.085$ so $T_{L}=1.585, t_{H}^{P}=0.54, t_{H}^{D}=1.25$ so $T_{H}=1.79$, and $\mu=1.04$. Under these assumptions, we have: $x_{L}^{*}=2.585, \widehat{x}=2.699, x_{H}^{*}=2.721, \widetilde{x}=3$, and $\mathcal{C}_{\{H L\}}^{*}=0.3259<\mathcal{C}_{\{L\}}^{*}=0.3288<$ $\mathcal{C}_{\{H\}}^{*}=0.3295$. In this example, all plaintiffs have weak cases as $\widetilde{x}=b$, yet $86 \%$ of them invest in case preparation when both technologies are available. This excessive investment entails inefficient sunk costs, but comes with a higher settlement rate. The settlement probability is indeed $98 \%$ in $\{H L\}$ as opposed to $79 \%$ only in $\{L\}$. Accordingly, trial costs are reduced, which more than offsets the increase in preparation costs.

\section{Concluding remarks}

The above presentation has assumed that the informed party is the plaintiff. The model, however, allows for alternative interpretations. Consider for instance the following tax 
evasion situation, where the informed party is the defendant. After a preliminary inspection, the tax department has found that an agent (firm or individual) hid some transactions and that a certain amount of taxes has not been paid as a result. Yet thanks to skillful accounting practices, the agent can justify a fraction of this tax evasion. The amount the agent can justify is his private information. Once challenged by the authorities, the agent can hire a costly tax advisor, who is able to reduce the tax liability even further. After observing this choice, the tax department makes a settlement offer to save on inspection costs. If the settlement is rejected by the agent, a thorough inspection starts which is costly for both the tax department and the taxpayer.

The model also applies to the following procurement issue. A firm or a government buys an input (e.g. a commodity) whose quality is variable. The price contractually depends on the estimated quality, but the quality audit is costly. To save on the evaluation costs, the buyer proposes a price to the supplier. If the latter refuses the proposed price, the audit is undertaken, and both the buyer and the supplier incur costs. The supplier can hire an engineer in charge of quality management, which entails a sunk cost but increases quality. The buyer makes her price offer after observing the supplier's effort in quality management.

More generally, the model applies to any context where a monetary transfer must be decided on the basis of unobserved characteristics of one party, and these characteristics can be revealed through a costly audit. To save on the auditing costs, the uninformed party makes an offer, but the informed party has the opportunity to move first, by investing to improve its position should the audit occur. Notice that the investment of the informed party could directly enhance the welfare through a real effect on attributes valued by the players (e.g. a quality improvement). In this paper, we have ruled out this possibility to focus exclusively on the signaling mechanism and the bluff effect.

We have assumed that the informed party initiates the case, and makes his preparation decision before the opposite party can move. Depending on the circumstances, however, the uninformed party may be able to anticipate future litigation and to make an initial offer at the very beginning of the process. Accordingly, we could envisage successive sequences of investment decisions and settlement offers. It would be of interest to study the resulting dynamics, in the spirit of Spier (1992).

Finally, an obvious limitation of our framework is that we study the strategic effects of case preparation of one party only, leaving the pretrial efforts of the adversary exogenous. It is tempting to allow both parties to invest. Under symmetric information, this could result in an arms race. Depending on the costs and returns of the respective investments, both parties could invest and neutralize each other -a prisoner dilemma. The current framework emphasizes a different idea: plaintiffs with weak cases invest to manipulate the beliefs of the opposite party; overinvestment is inherent to asymmetric information.

\section{References}

Lucian Arye Bebchuk. Litigation and settlement under imperfect information. RAND Journal of Economics, 15(3):404-15, Autumn 1984.

Bernard Black, Charles Silver, David A. Hyman, and William M. Sage. Stability, not crisis: 
Medical malpractice claims in texas, 1988-2002. Journal of Empirical Legal Studies, 2: 207-245, 2005.

Amitabh Chandra, Nundy Shantanu, and Seth A. Seabury. The growth of physician malpractice payments: Evidence from the national practitioner data bank. Health Affairs, 24:240-50, 2005.

Andrew F. Daughety and Jennifer F. Reinganum. Settlement negociations with two-sided asymmetric information: Model duality, information distribution, and efficiency. International Review of Law and Economics, 14:283-298, 1994.

Andrew F. Daughety and Jennifer F. Reinganum. Hush money. RAND Journal of Economics, 30(4):661-78, Winter 1999.

Andrew F. Daughety and Jennifer F. Reinganum. Economic theories of settlement bargaining. Annual Review of Law and Social Sciences, (1):35-59, 2005.

Bruce L. Hay. Effort, information, settlement, trial. Journal of Legal Studies, 24(1):29-62, January 1995.

David S. Kaplan, Joyce Sadka, and Jorge Luis Silva-Mendez. Litigation and settlement: New evidence from labor courts in mexico. forthcoming in the Journal of Empirical Legal Studies, 2008.

Lisa L. Posey. The plaintiff's attorney in the liability insurance claims settlement process: A game theoretic approach. Journal of Insurance Issues, 21(2):119-137, Fall 1998.

Jennifer F. Reinganum and Louis L. Wilde. Settlement, litigation, and the allocation of litigation costs. RAND Journal of Economics, 17(4):557-66, Winter 1986.

Joel L. Schrag. Managerial judges: An economic analysis of the judicial management of legal discovery. RAND Journal of Economics, 30(2):305-23, Summer 1999.

Urs Schweizer. Litigation and settlement under two-sided incomplete information. Review of Economic Studies, 56(2):163-77, April 1989.

Kathryn E. Spier. The dynamics of pretrial negotiation. Review of Economic Studies, 59 (1):93-108, January 1992.

Kathryn E. Spier. Litigation. forthcoming in the Handbook of Law and Economics, A. Mitchell Polinsky and Steven Shavell, Editors, 2005. 


\section{Appendix}

\section{A Proof of Lemma 1}

We have already shown that if $P_{e}$ is a probability distribution on $[a, b]$, then the functions $v_{H}$ and $v_{L}$ given (4) belongs to $K_{H}$ and $K_{L}$. Conversely, pick any $v_{L} \in K_{L}$. Integrating by parts and using $v_{L}(b)=b-t_{L}^{P}$, we have

$$
\begin{aligned}
v_{L}(x)= & -\int_{x}^{b} v_{L}^{\prime}(y) \mathrm{d} y+b-t_{L}^{P} \\
= & \int_{x}^{b} v_{L}^{\prime \prime}(y) \cdot\left[y-t_{L}^{P}\right] \mathrm{d} y+\left[1-v_{L}^{\prime}(b)\right] \cdot\left[b-t_{L}^{P}\right]+v_{L}^{\prime}(x) \cdot\left[x-t_{L}^{P}\right] \\
= & \int_{x}^{b} v_{L}^{\prime \prime}(y) \cdot\left[y-t_{L}^{P}\right] \mathrm{d} y+\int_{a}^{x} v_{L}^{\prime \prime}(y) \cdot\left[x-t_{L}^{P}\right] \mathrm{d} y \\
& +\left[1-v^{\prime}(b)\right] \cdot\left[b-t_{L}^{P}\right]+v_{L}^{\prime}(a)\left[x-t_{L}^{P}\right] \\
= & \int_{a}^{b} v_{L}^{\prime \prime}(y) \max \left\{x-t_{L}^{P}, y-t_{L}^{P}\right\} \mathrm{d} y \\
& +\left[1-v_{L}^{\prime}(b)\right]\left(b-t_{L}^{P}\right)+\left[x-t_{L}^{P}\right] v_{L}^{\prime}(a)
\end{aligned}
$$

The same computation yields

$$
\begin{aligned}
v_{H}(x)= & \int_{a}^{b} \frac{v_{H}^{\prime \prime}(y)}{\mu} \max \left\{\mu x-t_{H}^{P}, \mu y-t_{H}^{P}\right\} \mathrm{d} y \\
& +\left[1-\frac{v_{H}^{\prime}(b)}{\mu}\right]\left(\mu b-t_{H}^{P}\right)+\left[\mu x-t_{H}^{P}\right] \frac{v_{H}^{\prime}(a)}{\mu}-c .
\end{aligned}
$$

It follows that any $v_{e} \in K_{e}$ can be written according to (4) with $P_{e}$ given by

$$
\begin{aligned}
P_{L}(y) & =v_{L}^{\prime}(a) \delta_{a}+v_{L}^{\prime \prime}(y)+\left[1-v_{L}^{\prime}(b)\right] \delta_{b} \\
P_{H}(y) & =\frac{v_{H}^{\prime}(a)}{\mu} \delta_{a}+\frac{v_{H}^{\prime \prime}(y)}{\mu}+\left[1-\frac{v_{H}^{\prime}(b)}{\mu}\right] \delta_{b},
\end{aligned}
$$

where $\delta_{x}$ represents the mass point at $x$. Since $v_{e}$ is a convex function, its first-order derivative is a nondecreasing function and its second-order derivative is a positive measure. We have

$$
\int_{a}^{b} \mathrm{~d} P_{L}(y)=v_{L}^{\prime}(a)+\int_{a}^{b} v_{L}^{\prime \prime}(y) \mathrm{d} y+1-v_{L}^{\prime}(b)=1,
$$

so the total mass of $P_{L}$ is 1 . The same result holds for $P_{H}$. So $P_{L}$ and $P_{H}$ are probability distributions on $[a, b]$.

Notice that $P_{e}$ could, in theory, have mass points at $a$, at $b$ and at any interior point in $(a, b)$. An interior mass point corresponds, as already mentioned, to a convex kink of $v_{e}$ (jump of $v_{e}^{\prime}$, mass in $v_{e}^{\prime \prime}$ ). 


\section{B Proof of Lemma 2}

Integrating twice by parts and using the fact that $\pi_{L}(b)=-\left(b-t_{L}^{P}\right)$, we find that the defendant's profit, when she faces $e=L$, is given by

$$
\begin{aligned}
\int_{a}^{b}\left[-v_{L}(x)-T_{L} v_{L}^{\prime}(x)\right] f_{L}(x) \mathrm{d} x & =\int_{a}^{b} v_{L}^{\prime}(x)\left[F_{L}(x)-T_{L} f_{L}(x)\right] \mathrm{d} x-v_{L}(b) \\
& =-\int_{a}^{b} v_{L}^{\prime}(x) \pi_{L}^{\prime}(x)-\left[b-t_{L}^{P}\right] \\
& =\int_{a}^{b} \pi_{L}(x) v_{L}^{\prime \prime}(x) \mathrm{d} x-v_{L}^{\prime}(b) \pi_{L}(b)+v_{L}^{\prime}(a) \pi_{L}(a)-\left[b-t_{L}^{P}\right] \\
& =\int_{a}^{b} \pi_{L}(x) v_{L}^{\prime \prime}(x) \mathrm{d} x+\left[1-v_{L}^{\prime}(b)\right] \pi_{L}(b)+v_{L}^{\prime}(a) \pi_{L}(a) \\
& =\int_{a}^{b} \pi_{L}(x) \mathrm{d} P_{L}(x) .
\end{aligned}
$$

Similarly, the defendant's profit, when she faces $e=H$, is given by (use $\pi_{H}(b)=-\left(\mu b-t_{H}^{P}\right)$ )

$$
\begin{aligned}
\int_{a}^{b} & {\left[-v_{H}(x)-\frac{T_{H}}{\mu} v_{H}^{\prime}(x)\right] f_{H}(x) \mathrm{d} x-c=} \\
& =\int_{a}^{b} v_{H}^{\prime}(x)\left[F_{H}(x)-\frac{T_{H}}{\mu} f_{H}(x)\right]-v_{H}(b)-c \\
& =\frac{1}{\mu}\left(\int_{a}^{b} \pi_{H}(x) v_{H}^{\prime \prime}(x) \mathrm{d} x-v_{H}^{\prime}(b) \pi_{H}(b)+v_{H}^{\prime}(a) \pi_{H}(a)\right)-\left[\mu b-t_{H}^{P}\right] \\
& =\int_{a}^{b} \pi_{H}(x) v_{H}^{\prime \prime}(x) / \mu \mathrm{d} x+\left[1-v_{H}^{\prime}(b) / \mu\right] \pi_{H}(b)+\pi_{H}(a) v_{H}^{\prime}(a) / \mu \\
& =\int_{a}^{b} \pi_{H}(x) \mathrm{d} P_{H}(x) .
\end{aligned}
$$

\section{Technical properties of the equilibrium}

Lemma C.1. Suppose that the plaintiff chooses technology e with positive probability. Let $x \geq a$ be such $F_{e}(x)=0$. Then the defendant makes no offer smaller than or equal to $x$ after e: $P_{e}=v_{e}^{\prime}=0$ on $[a, x]$.

Proof. We write the proof for $e=L$. Suppose $\pi_{L}$ attains its maximum at $x=a$. This would imply

$$
\int_{a}^{x}\left[-F_{L}(x)+T_{L} f_{L}(x)\right] \mathrm{d} x \leq 0
$$

for all $x \in[a, b]$, or, noting $G_{L}=\int_{a}^{x} F_{L}(x) \mathrm{d} x$

$$
e^{-x / T_{L}}\left[-\frac{1}{T_{L}} G_{L}(x)+F_{L}(x)\right] \leq 0 .
$$


The function $e^{-x / T_{L}} G_{L}$ would be nonincreasing. Since it is zero at $x=a$, it would be everywhere nonpositive, which implies that $F_{L}$ and $f_{L}$ would be identically 0 . This contradicts the fact that $F_{L}$ is a probability distribution on $[a, b]$.

Lemma C.2. Let $x \in(a, b)$ be such that $\pi_{e}$ is not maximal at $x$. Then $v_{e}$ is affine on a neighborhood of $x$. Convex kinks in $v_{e}$ can occur only at points where $\pi_{e}$ attains its maximum.

Proof. Since $\pi_{e}$ is continuous, it is not maximal in a neighborhood of $x$, which, therefore, does no intersect the support of $P_{e}$. Since, in $(a, b)$, the support of $P_{e}$ is the same as the support of $v_{e}^{\prime \prime}$ (see Appendix A), $\pi_{e}$ is affine on that neighborhood.

Lemma C.3. If $x$ belongs to the support of $P_{H}$, then $\sigma\left(x^{+}\right) \leq \sigma\left(x^{-}\right)$. If $x$ belongs to the support of $P_{L}$, then $\sigma\left(x^{+}\right) \geq \sigma\left(x^{-}\right)$. If $x$ belongs to both supports, then $\sigma$ is continuous at $x$.

Proof. The result follows immediately from the fact that if $x$ maximizes $\pi_{e}$, then we must have: $\pi_{e}^{\prime}\left(x^{-}\right) \geq \pi_{e}^{\prime}\left(x^{+}\right)$.

\section{Proof of Proposition 1 (uniqueness part)}

The proof of the uniqueness of the equilibrium configuration when $x_{H}^{*}>\widetilde{x}$ requires a preliminary result.

Lemma D.1. Let $E$ be an equilibrium such that $v_{L}(\widetilde{x})=\widetilde{x}-t_{L}^{P}$. Let $\check{E}$ be the same configuration as $E$ except that $v_{H}$ is replaced by $\max \left(v_{L}, v_{H}\right)$ on $[a, \widetilde{x}]$.

Then $\check{E}$ is an equilibrium. For both litigants, the payoffs are the same at $E$ and $\check{E}$.

Proof. We have $v_{H}(\widetilde{x}) \geq v_{L}(\widetilde{x})=\widetilde{x}-t_{L}^{P}$, which yields: $\check{v}_{H}(\widetilde{x})=v_{H}(\widetilde{x})$. It follows immediately that $\check{v}_{H}$ belongs to $K_{H}$ and that the change corresponds to an admissible defendant's strategy $\check{P}_{H}$. The only impact of the change is the following: the plaintiffs who strictly preferred $L$ to $H$ at $E$ are indifferent between the two levels of effort at $\check{E}$. Those plaintiffs can therefore continue to choose $e=L$ with certainty ( $\check{\sigma}=\sigma=0$ is optimal for them).

We now use Lemma 2 to show that the defendant's profit is maximal at $\check{E}$. Since $\check{v}_{l}=v_{L}$ and the plaintiff's strategy is the same at $E$ and $\check{E}(\check{\sigma}=\sigma)$, the defendant's payoff when $e=L$ is the same at $E$ and $\check{E}$. Therefore the strategy $v_{L}$ still maximizes the defendant's payoff when $e=L$.

If the plaintiff invests in case preparation, the utility $v_{H}$ changes only when $\sigma=0$, so the integral $\int_{a}^{b} v_{H}(x) f_{H}(x) \mathrm{d} x$ is not affected. The derivatives $v_{H}^{\prime}$ and $\check{v}_{H}^{\prime}$ coincide whenever $v_{L} \neq v_{H}$, but can be different at points where $v_{H}=v_{L}$ and $\sigma=\check{\sigma}>0$ (corresponding to plaintiffs who randomize between the two technologies). The difference between $v_{H}^{\prime}$ and $\breve{v}_{H}^{\prime}$ at points where $f_{H}$ is positive could, in principle, affect the defendant's payoff. In fact, this is not the case, since such a possibility can only occur on a negligible set.

Indeed, $v_{H}$ and $v_{L}$ are differentiable almost everywhere. If $v_{H}(x)=v_{L}(x)$ and $v_{H}^{\prime}(x)=$ $v_{L}^{\prime}(x)$, then $\check{v}_{H}^{\prime}(x)=v_{H}^{\prime}(x)$, so $v_{H}^{\prime}$ and $\check{v}_{H}^{\prime}$ coincide. If $v_{H}(x)=v_{L}(x)$ and $v_{H}^{\prime}(x) \neq v_{L}^{\prime}(x)$, then $\breve{v}_{H}$ is not differentiable at $x$, which can occur only a negligible subset. 
It follows that $v_{H}^{\prime}$ and $\check{v}_{H}^{\prime}$ coincide at almost every $x$ such that $\sigma>0$ and that the defendant's payoff when $e=H$ is the same at $E$ and $\check{E}$. We conclude that the change in the defendant's strategy (from $v_{H}$ to $\check{v}_{H}$ ) does not affect her payoff.

To prove uniqueness, we proceed by contradiction. We suppose that there exists an equilibrium where the probability of $e=L$ is positive. From Lemma 3, we know that $v_{L}(\widetilde{x})=\widetilde{x}-t_{L}^{P}$. Lemma D.1 applies: there exists another equilibrium with the same plaintiff's strategy, the same payoff for both litigants and $v_{H} \geq v_{L}$ on $[a, \widetilde{x}]$. We now work with this equilibrium and exhibit a contradiction. Since, by assumption, the probability of $e=L$ is positive and given that high types $(x>\widetilde{x})$ chooses $e=H$ (recall Corollary 1 ), we cannot have $v_{H}>v_{L}$ everywhere on $(a, \widetilde{x}]$. Let $x_{1}, a<x_{1} \leq \widetilde{x}$, be the highest solution to $v_{L}=v_{H}$. By construction, all plaintiffs with type $x>x_{1}$ strongly prefer $e=H$ to $e=L$; in other words: $v_{H}>v_{L}$ and $\sigma=1$ on $\left(x_{1}, b\right]$. It follows that $\sigma\left(x_{1}^{+}\right)=1$.

We now use the property $v_{H} \geq v_{L}$ on $[a, \widetilde{x}]$ (coming from Lemma D.1) to prove that $x_{1}$ necessarily belongs to the support of $P_{H}$. Here again, we proceed by contradiction. Suppose that $x_{1}$ were not in the support of $P_{H}$. Then, by Lemma C.2, $v_{H}$ would be affine in a small interval around $x_{1}$. The function $v_{L}$ would be below this affine function for $x \leq x_{1}$, would coincide with it at $x_{1}$ and would be strictly below for $x>x_{1}$. This would violate the convexity of $v_{L}$. We conclude that $x_{1}$ belongs to the support of $P_{H}$.

Yet we have:

$$
\begin{aligned}
-\int_{a}^{x_{1}} \sigma(t) \mathrm{d} F(t)+\frac{T_{H}}{\mu} \sigma\left(x_{1}^{+}\right) f\left(x_{1}\right) & =-\int_{a}^{x_{1}} \sigma(t) \mathrm{d} F(t)+\frac{T_{H}}{\mu} f\left(x_{1}\right) \\
& \geq-F\left(x_{1}\right)+\frac{T_{H}}{\mu} f\left(x_{1}\right) \\
& =f\left(x_{1}\right) \cdot\left[T_{H} / \mu-\tau\left(x_{1}\right)\right] \\
& \geq f\left(x_{1}\right) \cdot\left[T_{H} / \mu-\tau(\widetilde{x})\right] \\
& >0 .
\end{aligned}
$$

This implies that $\pi_{H}^{\prime}\left(x_{1}^{+}\right)>0$, which contradicts $x_{1} \in \operatorname{supp} P_{H} \subset \operatorname{argmax} \pi_{H}$. This contradiction yields uniqueness.

\section{E Proof of Proposition 2}

We assume that $T_{H} / \mu<\tau(\widetilde{x})$ or $x_{H}^{*}<\widetilde{x}$. We already know that the defendant makes no offer greater than $\widetilde{x}$ after observing $L$, that is $v_{L}(\widetilde{x})=\widetilde{x}-t_{L}^{P}$. In this appendix, we show that, after observing $e=H$, the defendant makes no offer greater than $\mu \widetilde{x}-t_{H}^{P}$ with positive probability, which is equivalent to $v_{H}(\widetilde{x})=v_{L}(\widetilde{x})$.

We proceed by contradiction. Suppose that the defendant makes an offer $\mu x_{2}-t_{H}^{P}$, $\widetilde{x}<x_{2} \leq b$, with positive probability. We follow the same argument as in the proof of the uniqueness part of Proposition 1 . Since $v_{L}(\widetilde{x})=\widetilde{x}-t_{L}^{P}$, we can apply Lemma D.1: replacing 
$v_{H}$ by $\max \left(v_{L}, v_{H}\right)$ on $[a, \widetilde{x}]$, leaving $v_{L}$ and $\sigma$ unchanged, we get another equilibrium where both litigants get the same payoff. We now work with this new equilibrium and exhibit a contradiction. Since, by assumption, the probability of $L$ is positive and given that high types $(x>\widetilde{x})$ chooses $e=H$ by Corollary 1 , we cannot have $v_{H}>v_{L}$ everywhere on $(a, \widetilde{x}]$. Let $a<x_{1}<\widetilde{x}$ be the highest solution to $v_{L}=v_{H} \cdot{ }^{20}$ By construction, all plaintiffs with type $x>x_{1}$ strongly prefer $e=H$ to $e=L$; in other words: $v_{H}>v_{L}$ and $\sigma=1$ on $\left(x_{1}, b\right]$.

We now use the property (coming from Lemma D.1) that $v_{H} \geq v_{L}$ on $[a, \widetilde{x}]$, to prove that $x_{1}$ belongs to the support of $P_{H}$. Again, we proceed by contradiction. Suppose that $x_{1}$ were not in the support of $P_{H}$. Then, by Lemma C.2, $v_{H}$ would be affine in a small interval around $x_{1}$. Since $v_{L} \leq v_{H}$ on $[a, \widetilde{x}]$, the function $v_{L}$ would be below this affine function for $x \leq x_{1}$, would coincide with it at $x_{1}$ and would be strictly below for $x>x_{1}$. This would violate the convexity of $v_{L}$. We conclude that $x_{1}$ must belong to the support of $P_{H}$.

On $\left[x_{1}, b\right]$, we have $\sigma=1$ and $\pi_{H}^{\prime}$ is equal (up to a positive multiplicative factor) to

$$
-\int_{a}^{x} \sigma(t) f(t) \mathrm{d} t+\frac{T_{H}}{\mu} f(x)=\kappa-F(x)+\frac{T_{H}}{\mu} f(x)
$$

where $\kappa=\int_{a}^{x_{1}}(1-\sigma) \mathrm{d} F>0$. Since $\tau$ is nondecreasing, the function $\pi_{H}$ on $\left[x_{1}, b\right]$ is first nondecreasing, then nonincreasing: it attains its maximum at some point(s) greater than $x_{H}^{*}$. We must therefore have: $x_{H}^{*}<x_{1}<\widetilde{x}<x_{2}$. Since the function $\pi_{H}$ is maximal at $x_{1}$ and at $x_{2}$, it must remain constant in between, which implies $\kappa-F(x)+\frac{T_{H}}{\mu} f(x)=0$, or

$$
\frac{T_{H}}{\mu} \frac{1}{\tau}=1-\frac{\kappa}{F}
$$

on $\left[x_{1}, x_{2}\right]$. Since the left-hand side is decreasing and the right-hand side is increasing, it follows that both sides are constant, which implies that $F$ is constant, and $f=0$ on $\left[x_{1}, x_{2}\right]$, the desired contradiction. It follows that $v_{L}(\widetilde{x})=v_{H}(\widetilde{x})$.

\section{F $\quad$ Proof of Proposition 3}

The proof is organized as follows. First, we show that after $e=H$, the defendant offers $\mu \widetilde{x}-t_{H}^{P}$ with probability $1-1 / \mu$. Second, we establish a number of properties of offers made after $e=L$. Third, we exploit these properties to prove that, after $e=L$, the defendant makes only one offer (the support of $P_{L}$ is the singleton). Finally, noting $\widehat{x}-t_{L}^{P}$ this single offer, we show that the indifferent plaintiff $\widehat{x}$ uses a non-degenerated mixed strategy: $0<\sigma(\widehat{x})<1$.

\section{F.1 After $e=H$, the defendant offers $\mu \widetilde{x}-t_{H}^{P}$ with probability $1-1 / \mu$}

Since $v_{H}=v_{L}$ on $[a, \widetilde{x}]$ and $v_{L} \in K_{L}$, the left-derivative of $v_{H}$ at $\widetilde{x}$ is lower than or equal to 1 . Since $v_{H}^{\prime}\left(\widetilde{x}^{+}\right)=\mu$, the derivative of $v_{H} / \mu$ jumps upwards, with a jump greater than

\footnotetext{
${ }^{20}$ Note that, in the proof of the uniqueness part of Proposition 1 , we only knew that $x_{1} \leq \widetilde{x}$.
} 
or equal to $1-1 / \mu$. The probability of playing $\widetilde{x}$ after $e=H$ has to be greater (or equal to) than $1-1 / \mu$.

It follows that $\pi_{H}$ is maximal at $\widetilde{x}$. This implies that $\sigma$ is continuous at $\widetilde{x}$. Indeed, if $\sigma\left(\widetilde{x}^{-}\right)$were strictly smaller than $\sigma\left(\widetilde{x}^{+}\right)=1$, we would have $\pi_{H}^{\prime}\left(\widetilde{x}^{-}\right)<\pi_{H}^{\prime}\left(\widetilde{x}^{+}\right)=0$ and $\pi_{H}$ would not be maximal at $\widetilde{x}$.

Since $-F_{L}(\widetilde{x})+T_{L} f_{L}(\widetilde{x})=-F_{L}(\widetilde{x})<0, \pi_{L}$ is not maximal at $\widetilde{x}$ and $v_{L}$ is affine on a neighborhood of $\widetilde{x}$. This implies $v^{\prime}\left(\widetilde{x}^{-}\right)=1$. So the jump of $v_{H}^{\prime} / \mu$ at $\widetilde{x}$ is exactly $1-1 / \mu$. We conclude that, after $e=H$, the defendant offers $\mu \widetilde{x}-t_{H}^{P}$ with probability $1-1 / \mu$

\section{F.2 Properties of offers made after $e=L$}

By Lemma 3, we know that any offer made after $e=L$, say $\widehat{x}-t_{L}^{P}$, satisfies: $\widehat{x} \leq \widetilde{x}$. As mentioned above, $\pi_{L}$ is not maximal at $\widetilde{x}$, so we have: $\widehat{x}<\widetilde{x}$. From Proposition 2 , we know that $v_{H}=v_{L}$ on $[a, \widetilde{x}]$. From Lemma 1, we deduce that the supports of $P_{L}$ and $P_{H}$ coincide on this interval. It follows that $\widehat{x}$ belongs to the support of both $P_{H}$ and $P_{L}$, and that the functions $\pi_{H}$ and $\pi_{L}$ both attain their maximum at $\widehat{x}$. From Lemma C.3, $\sigma$ must be continuous at this point, and $\pi_{H}$ and $\pi_{L}$ are differentiable at $\widehat{x}$, so we have

$$
\begin{aligned}
\int_{a}^{\widehat{x}} \sigma \mathrm{d} F & =\frac{T_{H}}{\mu} \sigma(\widehat{x}) f(\widehat{x}) \\
\int_{a}^{\widehat{x}}(1-\sigma) \mathrm{d} F & =T_{L}[1-\sigma(\widehat{x})] f(\widehat{x}) .
\end{aligned}
$$

Adding up this two equations yields

$$
F(\widehat{x})=\left[T_{H} / \mu-T_{L}\right] \sigma(\widehat{x}) f(\widehat{x})+T_{L} f(\widehat{x}),
$$

which implies $\widehat{x}=x_{H}^{*}=x_{L}^{*}$ when $T_{H} / \mu=T_{L}$, and equation (15) otherwise:

$$
\sigma(\widehat{x})=\frac{\tau(\widehat{x})-T_{L}}{T_{H} / \mu-T_{L}}
$$

As the probability $\sigma(\widehat{x})$ is between 0 and 1 , and as $\tau$ is increasing, it follows that $\widehat{x}$ lies between $x_{L}^{*}$ and $x_{H}^{*}$. More precisely, if $T_{L}<T_{H} / \mu$, then $x_{L}^{*}<\widehat{x}<x_{H}^{*}$ and if $T_{H} / \mu<T_{L}$, then $x_{H}^{*}<\widehat{x}<x_{L}^{*}$ (see Figures $6 \mathrm{a}$ and $6 \mathrm{~b}$ ).

Since $\pi_{H}$ attains its maximum at $\widehat{x}$, we have

$$
\int_{\widehat{x}}^{x}\left[-F_{H}(y)+\frac{T_{H}}{\mu} f_{H}(y)\right] \mathrm{d} y \leq 0
$$

for all $y$. Setting $G_{H}(x)=\int_{\widehat{x}}^{x} F_{H}(y) \mathrm{d} y$, we get

$$
-G_{H}(x)+\frac{T_{H}}{\mu} F_{H}(x) \leq \frac{T_{H}}{\mu} F_{H}(\widehat{x}) .
$$

Multiplying by $\exp \left(-\mu x / T_{H}\right)$ yields

$$
\exp \left(-\mu x / T_{H}\right)\left[-\frac{\mu}{T_{H}} G_{H}(x)+F_{H}(x)\right] \leq F_{H}(\widehat{x}) \exp \left(-\mu x / T_{H}\right) .
$$


Integrating between $\widehat{x}$ and $x \geq \widehat{x}$ yields

$$
G_{H}(x) \leq \frac{T_{H}}{\mu} F_{H}(\widehat{x})\left[\exp \left(\frac{\mu}{T_{H}}(x-\widehat{x})\right)-1\right]
$$

Now using (16) yields, after simplification

$$
F_{H}(x) \leq F_{H}(\widehat{x}) \exp \left[\frac{\mu}{T_{H}}(x-\widehat{x})\right]
$$

for every $x \geq \widehat{x}$. The same computation for the $L$-technology shows that

$$
F_{L}(x) \leq F_{L}(\widehat{x}) \exp \left[\frac{1}{T_{L}}(x-\widehat{x})\right]
$$

\section{F.3 After $e=L$, the defendant makes only one offer}

We now prove that $P_{L}$ is a mass point. We proceed by contradiction. We assume that there exists $x_{1}$ and $x_{2}, a<x_{1}<x_{2}<\widetilde{x}$, both in the supports of $P_{L}$ and $P_{H}$.

If $T_{H} / \mu=T_{L}$, we already know that this is impossible, since we must have $x_{1}=x_{2}=$ $x_{H}^{*}=x_{L}^{*}$. We assume $T_{L}<T_{H} / \mu$, which implies (see the preceding section): $x_{L}^{*}<x_{1}<$

$x_{2}<x_{H}^{*} \cdot{ }^{21}$ Applying (17) for $\widehat{x}=x_{1}$ and $x=x_{2}$ we obtain (using the f.o.c. (13) for both $x_{1}$ and $\left.x_{2}\right)$ :

$$
\sigma\left(x_{2}\right) f\left(x_{2}\right) \leq \sigma\left(x_{1}\right) f\left(x_{1}\right) \exp \left[\frac{\mu}{T_{H}}\left(x_{2}-x_{1}\right)\right] .
$$

From (15), we have for $i=1,2$ :

$$
\sigma\left(x_{i}\right) f\left(x_{i}\right)=\frac{F\left(x_{i}\right)-T_{L} f\left(x_{i}\right)}{T_{H} / \mu-T_{L}}>0
$$

and as $T_{L}<T_{H} / \mu, F\left(x_{i}\right)-T_{L} f\left(x_{i}\right)>0$. Therefore, inequality (19) can be rewritten as

$$
F\left(x_{2}\right)-T_{L} f\left(x_{2}\right) \leq\left(F\left(x_{1}\right)-T_{L} f\left(x_{1}\right)\right) \exp \left[\frac{\mu}{T_{H}}\left(x_{2}-x_{1}\right)\right]
$$

or taking the logarithm

$$
\log \left(F\left(x_{2}\right)-T_{L} f\left(x_{2}\right)\right)-\log \left(F\left(x_{1}\right)-T_{L} f\left(x_{1}\right)\right) \leq \frac{\mu}{T_{H}}\left(x_{2}-x_{1}\right) .
$$

Let $\Lambda(x)=\ln \left(F(x)-T_{L} f(x)\right)=\ln F(x)+\ln \left(1-T_{L} / \tau(x)\right)$. Since $F$ is log-concave and $\tau$ is increasing, we have $\tau\left(x_{2}\right)<\tau\left(x_{H}^{*}\right)=T_{H} / \mu$, and

$$
\Lambda\left(x_{2}\right)-\Lambda\left(x_{1}\right) \geq \ln F\left(x_{2}\right)-\ln F\left(x_{1}\right) \geq \frac{1}{\tau\left(x_{2}\right)} .\left(x_{2}-x_{1}\right) \geq \frac{\mu}{T_{H}}\left(x_{2}-x_{1}\right),
$$

which, combined to (20), yields $x_{1}=x_{2}$, the desired contradiction. We must therefore have: $x_{1}=x_{2}$.

\footnotetext{
${ }^{21}$ The case $x_{H}^{*}<x_{1}<x_{2}<x_{L}^{*}$ is treated similarly starting with (18) instead of (17).
} 


\section{F.4 The plaintiff $\widehat{x}$ randomizes: $0<\sigma(\widehat{x})<1$}

We now prove that the probability $\sigma(\widehat{x})$ is strictly between 0 and 1 as it satisfies

$$
\frac{f(\widetilde{x})}{f(\widehat{x})} \exp \left[\frac{\mu}{T_{H}}(\widehat{x}-\widetilde{x})\right] \leq \sigma(\widehat{x}) \leq 1-\frac{\operatorname{Pr}(e=L)}{T_{L} f(\widehat{x})} \exp \left[\frac{1}{T_{L}}(\widehat{x}-\widetilde{x})\right],
$$

where $\operatorname{Pr}(e=L)=1-\operatorname{Pr}(e=H)$ is known from (11).

Indeed, using the first-order condition (13) and applying (17) at $x=\widetilde{x}$ yields

$$
F_{H}(\widetilde{x})=\frac{T_{H}}{\mu} \frac{f(\widetilde{x})}{\operatorname{Pr}(e=H)} \leq \frac{T_{H}}{\mu} \frac{\sigma(\widehat{x}) f(\widehat{x})}{\operatorname{Pr}(e=H)} \exp \left[\frac{\mu}{T_{H}}(\widetilde{x}-\widehat{x})\right]
$$

which yields the left inequality of (22). Using the first-order condition (14) and applying (18) at $x=\widetilde{x}$ yields

$$
F_{L}(\widetilde{x})=1 \leq T_{L} \frac{(1-\sigma(\widehat{x})) f(\widehat{x})}{\operatorname{Pr}(e=L)} \exp \left[\frac{1}{T_{L}}(\widetilde{x}-\widehat{x})\right]
$$

which yields the right inequality of (22).

\section{G The defendant's payoff}

Another way of writing the defendant's profit is to express it as the difference between the total welfare and the utility left to the plaintiff (Lemma 2). Given that $v_{L}=v_{H}=v^{*}$, we have:

$$
\begin{aligned}
-\Pi_{\{H L\}}^{*}= & -\operatorname{Pr}(e=L) \Pi_{L}^{*}-\operatorname{Pr}(e=H) \Pi_{H}^{*} \\
= & \int_{a}^{b}\left\{\left[v(x)+T_{L} v^{\prime}(x)\right][1-\sigma(x)]+\left[v(x)+\frac{T_{H}}{\mu} v^{\prime}(x)\right] \sigma(x)\right\} f(x) \mathrm{d} x \\
& +c \operatorname{Pr}(e=H) .
\end{aligned}
$$

Using equation (11) and observing that $v^{\prime}=1$ on $[\widehat{x}, \widetilde{x}]$ yields (12).

\section{H Construction of an equilibrium (Proof of Proposition 4)}

We examine successively the case $T_{H} / \mu<T_{L}, T_{H} / \mu=T_{L}$ and $T_{H} / \mu>T_{L}$. Hereafter, we adopt the natural notations: $f_{L}^{*}=f\left(x_{L}^{*}\right), F_{L}^{*}=F\left(x_{L}^{*}\right), f_{H}^{*}=f\left(x_{H}^{*}\right), F_{H}^{*}=F\left(x_{H}^{*}\right)$, $\tilde{f}=f(\widetilde{x}), \tilde{F}=F(\widetilde{x})$.

\section{H.1 The case $T_{H} / \mu<T_{L}$}

Using the log-concavity of $F$, we get, for $x_{H}^{*}<x_{1}<x_{2}<\widetilde{x}$ :

$$
\ln F\left(x_{2}\right)-\ln F\left(x_{1}\right) \leq \frac{1}{\tau\left(x_{1}\right)}\left(x_{2}-x_{1}\right) \leq \frac{\mu}{T_{H}}\left(x_{2}-x_{1}\right) .
$$


It follows that $-\ln F(x)+\frac{\mu}{T_{H}} x$ is nondecreasing on $\left[x_{H}^{*}, \widetilde{x}\right]$. Since $\tau=F / f$ is increasing, it follows that $-\ln f(x)+\frac{\mu}{T_{H}} x$ is increasing, and so is $\sigma^{*}$ on $\left[x_{H}^{*}, \widetilde{x}\right]$. Since $T_{H} / \mu-T_{L}<0$, the function $\left(\tau-T_{L}\right) /\left(T_{H} / \mu-T_{L}\right)$ is decreasing on $\left[x_{H}^{*}, \widetilde{x}\right]$, is equal to 1 at $x_{H}^{*}$ and to 0 at $x_{L}^{*}$. By continuity, the equation

$$
\sigma^{*}(x)=\frac{\tau(x)-T_{L}}{T_{H} / \mu-T_{L}}
$$

has a unique solution $\widehat{x}$ in $\left[x_{H}^{*}, \min \left(\widetilde{x}, x_{L}^{*}\right)\right]$. Notice that we have: $\sigma^{*} \geq\left(\tau-T_{L}\right) /\left(T_{H} / \mu-T_{L}\right)$ on $[\widehat{x}, \widetilde{x}]$.

We have: $\sigma^{*}(\widetilde{x})=1$, so $\sigma^{*}$ is continuous at $\widetilde{x}$. The function $\sigma^{*}$ takes its value in $(0,1]$ and is continuous on $[a, b]$. Since $\sigma^{*}$ is constant on $\left[a, x_{H}^{*}\right]$, we have have

$$
\begin{aligned}
\int_{a}^{x_{H}^{*}} \sigma^{*}(x) f(x) \mathrm{d} x=\frac{\tilde{f}}{f_{H}^{*}} \exp \left[\frac{\mu}{T_{H}}\left(x_{H}^{*}-\widetilde{x}\right)\right] \cdot F_{H}^{*} & =\frac{T_{H}}{\mu} \tilde{f} \exp \left[\frac{\mu}{T_{H}}\left(x_{H}^{*}-\widetilde{x}\right)\right] \\
& =\frac{T_{H}}{\mu} \sigma^{*}\left(x_{H}^{*}\right) f\left(x_{H}^{*}\right),
\end{aligned}
$$

which yields $\pi_{H}^{\prime}\left(x_{H}^{*}\right)=0$. From the definition of $\sigma^{*}$ on $\left(x_{H}^{*}, \widetilde{x}\right)$, it follows that $\pi_{H}^{\prime}$ is constant on that interval, which, given that $\pi_{H}^{\prime}\left(x_{H}^{*}\right)=0$, yields $\pi_{H}^{\prime}=0$ on that interval. On $\left[a, x_{H}^{*}\right]$, the quantity $-F_{H}+\frac{T_{H}}{\mu} f_{H}$ is equal, up to a multiplicative positive constant, to $-F+\frac{T_{H}}{\mu} f \geq 0$, which yields $\pi_{H}^{\prime} \geq 0$ on $\left[a, x_{H}^{*}\right]$. Finally, on $[\widetilde{x}, b]$, we have $\sigma^{*}=1$ and the quantity $-F_{H}+\frac{T_{H}}{\mu} f_{H}$ is equal, up to an additive constant, to $-F+\frac{T_{H}}{\mu} f$, which is decreasing on $[\widetilde{x}, b]$ (by log-concavity of $F$ ). This shows that $\pi_{H}^{\prime} \leq 0$ on this interval. In sum, the function $\pi_{H}$ is nondecreasing on $\left[a, x_{H}^{*}\right]$ and constant on $\left[x_{H}^{*}, \widetilde{x}\right]$ and nonincreasing (and concave) on $[x, b]$.

Using $\widehat{x}<x_{L}^{*}, \sigma$ constant, and the log-concavity of $F$, it is easy to check that $\pi_{L}$ is nondecreasing on $[a, \widehat{x}]$. On $[\widehat{x}, \widehat{x}]$, we have, by using $\pi_{H}^{\prime}=0$ :

$$
\begin{aligned}
-\int_{a}^{x}\left(1-\sigma^{*}(t)\right) f(t) \mathrm{d} t+T_{L}\left(1-\sigma^{*}(x)\right) f(x) & =-F(x)+\int_{a}^{x} \sigma^{*}(t) f(t) \mathrm{d} t+T_{L}\left(1-\sigma^{*}(x)\right) f(x) \\
& =-F(x)+\left(\frac{T_{H}}{\mu}-T_{L}\right) \sigma^{*}(x) f(x)+T_{L} f(x) \\
& =\left(T_{H} / \mu-T_{L}\right) f(x)\left[\sigma^{*}(x)-\frac{\tau(x)-T_{L}}{T_{H} / \mu-T_{L}}\right] \\
& \leq 0
\end{aligned}
$$

which yields $\pi_{L}^{\prime} \leq 0$. It follows that $\pi_{L}$ is nonincreasing on $[\widehat{x}, \widetilde{x}]$. On $[\widetilde{x}, b]$, we have $\sigma^{*}=1$, and $\pi_{L}$ is affine and decreasing. In sum, $\pi_{L}$ is nondecreasing on $[a, \widehat{x}]$ and nonincreasing on $[\widehat{x}, b]$.

We have shown that $\pi_{H}$ attains its maximum at $\widehat{x}$ and $\widetilde{x}$ and that $\pi_{L}$ attains its maximum at $\widehat{x}$. Therefore the corresponding strategy $\left(P_{H}, P_{L}\right)$ is optimal for the defendant. Any plaintiff with type below $\widetilde{x}$ is indifferent between investing or not, and may randomize according to the proposed probability $\sigma^{*}$. 


\section{H.2 The case $T_{H} / \mu=T_{L}$}

Using $\sigma$ constant and the log-concavity of $F$, it is easy to check that $\pi_{H}$ and $\pi_{L}$ are nondecreasing on $[a, \widehat{x}]$. As above, $\pi_{H}$ is constant on $\left[x_{H}^{*}, \widetilde{x}\right]$ and decreasing and concave on $[\widetilde{x}, b]$. On $[\widehat{x}, \widetilde{x}]$, the same computation as above shows that

$$
-\int_{a}^{x}\left(1-\sigma^{*}(t)\right) f(t) \mathrm{d} t+T_{L}\left(1-\sigma^{*}(x)\right) f(x)=-F(x)+T_{L} f(x)=-F(x)+\frac{T_{H}}{\mu} f(x) \leq 0,
$$

implying that $\pi_{L}$ is nonincreasing on $[\widehat{x}, \widetilde{x}]$. On $[\widetilde{x}, b], \pi_{L}$ is still affine and decreasing.

\section{H.3 The case $T_{H} / \mu>T_{L}$}

As shown above, the function $(\tilde{f} / f) \exp \left[\frac{\mu}{T_{H}}(x-\widetilde{x})\right]$ is increasing on $\left[x_{H}^{*}, \widetilde{x}\right]$, and is equal to 1 at $\widetilde{x}$. Thus the value of the function at $x_{H}^{*}$ is smaller than or equal 1 . The function $\left(\tau-T_{L}\right) /\left(T_{H} / \mu-T_{L}\right)$ is also increasing on $\left[x_{L}^{*}, x_{H}^{*}\right]$, is equal to 0 at $x_{L}^{*}$, to 1 at $x_{H}^{*}$ and is greater than 1 at $\widetilde{x}$. It follows that the equation

$$
\frac{\tilde{f}}{f(x)} \exp \left[\frac{\mu}{T_{H}}(x-\widetilde{x})\right]=\frac{\tau(x)-T_{L}}{T_{H} / \mu-T_{L}}
$$

has at least one solution in $\left[x_{L}^{*}, \min \left(x_{H}^{*}, \widetilde{x}\right)\right]$. We define $\widehat{x}<\widetilde{x}$ as the highest root in this interval.

By the same reasoning as above (using the log-concavity of $F$ ), we obtain that $\sigma^{*}$ is decreasing on $\left[x_{L}^{*}, \widehat{x}\right]$. Since $\sigma^{*}$ is constant on $\left[a, x_{L}^{*}\right], \sigma^{*}$ takes its values in $[0,1]$, and we have: $\sigma^{*} \geq\left(\tau-T_{L}\right) /\left(T_{H} / \mu-T_{L}\right)$ on $[a, \widehat{x}]$ and $\sigma^{*} \leq\left(\tau-T_{L}\right) /\left(T_{H} / \mu-T_{L}\right)$ on $[\widehat{x}, \widehat{x}]$.

We have

$$
\begin{aligned}
\int_{a}^{x_{L}^{*}}\left(1-\sigma^{*}(x)\right) f(x) \mathrm{d} x & =(1-\hat{\sigma})\left(\hat{f} / f_{L}^{*}\right) \cdot \exp \left[\frac{1}{T_{L}}\left(x_{L}^{*}-\widehat{x}\right)\right] \cdot F_{L}^{*} \\
& =T_{L}(1-\hat{\sigma}) \hat{f} \exp \left[\frac{1}{T_{L}}\left(x_{L}^{*}-\widehat{x}\right)\right] \\
& =T_{L}\left(1-\sigma\left(x_{L}^{*}\right)\right) f\left(x_{L}^{*}\right),
\end{aligned}
$$

which yields $\pi_{L}^{\prime}\left(x_{L}^{*}\right)=0$. Since, by construction of $\sigma^{*}, \pi_{L}^{\prime}$ is constant on $\left[x_{L}^{*}, \widehat{x}\right]$, we also have: $\pi_{L}^{\prime}=0$ on that interval. In particular: $\pi_{L}^{\prime}(\widehat{x})=0$. The definition of $\widehat{x}$ then implies: $\pi_{H}^{\prime}(\widehat{x})=0$. Now, by construction of $\sigma^{*}, \pi_{H}^{\prime}$ is constant on $[\widehat{x}, \widehat{x}]$, which, in turn, yields: $\pi_{H}^{\prime}=0$ on that interval. As in case (i) and (ii), we have $\sigma^{*}=1$ on $[\widetilde{x}, b]$, and $\pi_{H}$ is concave and nonincreasing on that interval.

Again, usign $\sigma$ constant on $\left[a, x_{L}^{*}\right]$, it is easy to check that $\pi_{L}$ and $\pi_{H}$ are nondecreasing 
on that interval. On $\left[x_{L}^{*}, \widehat{x}\right]$, we have, by using $\pi_{L}^{\prime}=0$ :

$$
\begin{aligned}
-\int_{a}^{x} \sigma^{*} f(t) \mathrm{d} t+\frac{T_{H}}{\mu} \sigma^{*}(x) f(x) & =-F(x)+\int_{a}^{x}\left(1-\sigma^{*}\right) f(t) \mathrm{d} t+\frac{T_{H}}{\mu} \sigma^{*}(x) f(x) \\
& =-F(x)+T_{L}\left(1-\sigma^{*}(x)\right) f(x)+\frac{T_{H}}{\mu} \sigma^{*}(x) f(x) \\
& =-F(x)+T_{L} f(x)+\sigma^{*}(x) f(x)\left(\frac{T_{H}}{\mu}-T_{L}\right) \\
& =f(x)\left(T_{H} / \mu-T_{L}\right)\left[\sigma^{*}(x)-\frac{\tau(x)-T_{L}}{T_{H} / \mu-T_{L}}\right] \\
& \geq 0,
\end{aligned}
$$

implying that $\pi_{H}$ is nondecreasing on $\left[x_{L}^{*}, \widehat{x}\right]$. By construction, $\pi_{H}$ is constant on $[\widehat{x}, \widetilde{x}]$, and is concave and nonincreasing on $[\widetilde{x}, b]$. It follows that $\pi_{H}$ attains its maximum at $\widehat{x}$ and $\widetilde{x}$.

As to $\pi_{L}$, we already know that it is nondecreasing on $\left[a, x_{L}^{*}\right]$ and constant on $\left[x_{L}^{*}, \widehat{x}\right]$. Now on $[\widehat{x}, \widetilde{x}]$, the same computation as in case (i) shows, by using $\pi_{H}^{\prime}=0$, that

$$
-\int_{a}^{x}\left(1-\sigma^{*}\right) f(t) \mathrm{d} t+T_{L}\left(1-\sigma^{*}(x)\right) f(x)=\left(T_{H} / \mu-T_{L}\right) f(x)\left[\sigma^{*}(x)-\frac{\tau(x)-T_{L}}{T_{H} / \mu-T_{L}}\right] \leq 0
$$

implying that $\pi_{L}$ is nonincreasing on $[\widehat{x}, \widetilde{x}]$. Finally on $[\widetilde{x}, b]$, we know that $\sigma^{*}=1$, so $\pi_{L}$ is affine and decreasing, which shows that $\pi_{L}$ attains its maximum at $\widehat{x}$.

\section{Proof of Proposition 5}

In this section, we prove that, when $T_{L} \leq x_{L}^{*}=x_{H}^{*}<\widetilde{x}$, the defendant prefers $\{H L\}$ to $\{H\}$. Corollary 5 yields the expected payoff of the defendant in equilibrium when both technologies are available:

$$
\Pi_{\{H L\}}^{*}=-\left(\widehat{x}-t_{L}^{P}\right) \int_{a}^{\widehat{x}} f(x) \mathrm{d} x-\int_{\widehat{x}}^{\widetilde{x}}\left[x+t_{L}^{D}\right] f(x) \mathrm{d} x-\int_{\widetilde{x}}^{b}\left[\mu x+t_{H}^{D}\right] f(x) \mathrm{d} x-T_{L} f(\widetilde{x}) c .
$$

When only the $H$ technology is available, the defendant's profit is

$$
\Pi_{\{H\}}^{*}=-\left(\mu \widehat{x}-t_{H}^{P}\right) \int_{a}^{\widehat{x}} f(x) \mathrm{d} x-\int_{\widehat{x}}^{b}\left[\mu x+t_{H}^{D}\right] f(x) \mathrm{d} x
$$

whence

$$
\begin{aligned}
\Pi_{\{H L\}}^{*}-\Pi_{\{H\}}^{*} & =\left(\mu \widehat{x}-t_{H}^{P}-\left(\widehat{x}-t_{L}^{P}\right)\right) \int_{a}^{\widehat{x}} f(x) \mathrm{d} x+(\mu-1) \int_{\widehat{x}}^{\widetilde{x}} x f(x) \mathrm{d} x-T_{L} f(\widetilde{x}) c \\
& +\left(t_{H}^{D}-t_{L}^{D}\right)(F(\widetilde{x})-F(\widehat{x})) \\
& =(\mu-1)\left(\widehat{x}-T_{L}\right) \int_{a}^{\widehat{x}} f(x) \mathrm{d} x+(\mu-1) \int_{\widehat{x}}^{\widetilde{x}} x f(x) \mathrm{d} x-T_{L} f(\widetilde{x}) c \\
& +\left(t_{H}^{D}-t_{L}^{D}\right) F(\widetilde{x})
\end{aligned}
$$


Using $\widetilde{x}=\left(t_{H}^{P}-t_{L}^{P}+c\right) /(\mu-1)=T_{L}+c /(\mu-1)-\left(t_{H}^{D}-t_{L}^{D}\right) /(\mu-1)$ and $F(\widetilde{x})>T_{L} f(\widetilde{x})$, we get

$$
\begin{aligned}
\frac{\Pi_{\{H L\}}^{*}-\Pi_{\{H\}}^{*}}{\mu-1} & \geq\left(\widehat{x}-T_{L}\right) F(\widehat{x})+\int_{\widehat{x}}^{\widetilde{x}} x f(x) \mathrm{d} x-T_{L} f(\widetilde{x})\left(\widetilde{x}-T_{L}\right) \\
& =\left(\widetilde{x}-T_{L}\right) F(\widetilde{x})+\int_{\widehat{x}}^{\widetilde{x}}\left[-F(x)+T_{L} f(x)\right] \mathrm{d} x-T_{L} f(\widetilde{x})\left(\widetilde{x}-T_{L}\right) \\
& =\int_{\widehat{x}}^{\widetilde{x}}\left[-F(x)+T_{L} f(x)\right] \mathrm{d} x+\left(\widetilde{x}-T_{L}\right)\left[F(\widetilde{x})-T_{L} f(\widetilde{x})\right]
\end{aligned}
$$

Using the log-concavity of $F$, it is easy to check that the function $-F+T_{L} f$ is nonincreasing on $[\widehat{x}, \widehat{x}]$, which yields (using the assumption $\widehat{x}=x_{L}^{*}>T_{L}$ )

$$
\begin{aligned}
\frac{\Pi_{\{H L\}}^{*}-\Pi_{\{H\}}^{*}}{\mu-1} & \geq(\widetilde{x}-\widehat{x})\left[-F(\widetilde{x})+T_{L} f(\widetilde{x})\right]+\left(\widetilde{x}-T_{L}\right)\left[F(\widetilde{x})-T_{L} f(\widetilde{x})\right] \\
& =\left(\widehat{x}-T_{L}\right)\left[F(\widetilde{x})-T_{L} f(\widetilde{x})\right] \\
& \geq 0 .
\end{aligned}
$$

